Ann. Biol. anim. Bioch. Biophys., I97I, 11 (I), 5-39.

\title{
CROISSANCE RELATIVE PONDÉRALE POSTNATALE, DANS LES DEUX SEXES, DES PRINCIPAUX TISSUS ET ORGANES DE L'AGNEAU MERINOS D'ARLES
}

\author{
M. BÉNÉVENT \\ avec la collaboration technique de C. Bressot et Suzanne Paolantonacci \\ Station de Physiologie animale, \\ Centre de Recherches de Montpellier, I. N. R. A., \\ École nationale supérieure agronomique, \\ 34-Montpellier \\ RÉSUMÉ
}

De nombreux travaux concernant la croissance relative chez l'Agneau se rapportent à des organes ou des tissus particuliers et sont difficiles à comparer du fait de conditions d'étude généralement dissemblables. C'est pourquoi nous avons tenté d'analyser, à des stades suffisamment rapprochés et dans des conditions de croissance bien définies, les particularités du développement relatif des principaux tissus et organes chez l'agneau Mérinos d'Arles.

A cet effet ont été entièrement disséqués 7 groupes de 4 animaux chacun ( 2 mâles et 2 femelles) échelonnés de la naissance à l'âge de i6o jours, au long d'une même courbe de croissance.

Les données pondérales obtenues ont été utilisées à différentes fins :

- calcul des coefficients d'allométrie durant les premiers stades postnataux;

- analogies et différences du développement relatif selon le sexe;

- étude comparée de la croissance relative des différentes parties de l'organisme;

- mise en évidence de stades critiques de la croissance et de certaines liaisons entre organes.

Ces résultats sont discutés et font éventuellement l'objet de différentes hypothèses.

\section{INTRODUCTION}

La croissance relative a fait l'objet de nombreuses études chez des animaux très divers, mais pour les mammifères domestiques notamment, la connaissance en demeure encore assez fragmentaire.

Chez le Mouton, les travaux fondamentaux de HAmmond (r932) en ont abordé de nombreux aspects. Ils s'appuient toutefois sur un nombre très réduit d'animaux, parfois de races différentes, et les premiers stades du développement postnatal, entre o et 3 mois, n'y apparaissent pas. Divers auteurs de l'école de Hammond ont encore 
précisé certains aspects de la croissance relative chez l'Agneau, dans différentes conditions, notamment : évolution de certains organes, mise en évidence de "gradients de croissance ", influence du niveau de nutrition (WALLACE, I948 ; PALSSON et Verges, I952 $a$ et I952 $b$; PAL,SSON, I955; McMeEKAN, I960). Mais les stades étudiés au cours de la croissance sont encore souvent peu nombreux et assez éloignés entre eux. Ces travaux sont en général orientés de préférence, et de nombreux autres uniquement (SEEBECK, I968), vers l'étude du développement relatif des trois principaux tissus de la carcasse, os, gras, muscles, en liaison avec l'estimation de la qualité bouchère. Cette dernière a d'ailleurs fait l'objet d'une mise au point bibliographique de FlamanT et Boccard (I966).

D'autres travaux sont plus partiels, se rapportant par exemple aux seules relations entre muscles et os (RUSSEL et BARTon, I967; JACKSON, I967) ou encore au seul tissu gras (Marcheilo et $a l$, ig6r ; Cramer et Marchello, ig64).

Parfois sont considérées différentes régions corporelles de 1'Agneau en croissance (Boccard, Dumont, LefFebvre, I962). Parfois aussi, en vue de certains objectifs particuliers de recherche, sont publiées des études de la croissance relative de divers organes, isolés ou non : organes viscéraux (WARDROP, COMBE, I960-I96I ; LARGE, I964), glande mammaire (WALIACE, I953), testicule (COUROT, I962), forme des côtes (PALSSON, VeRGES, I965).

Outre le fait que le plus grand nombre des travaux n'ont ainsi concerné qu'une partie déterminée de l'organisme, les études ont été réalisées dans des conditions d'élevage bien souvent différentes, sur des races diverses, sur un sexe ou un autre, et à des stades variables mais rarement correspondants. Ces conditions rendent évidemment les comparaisons difficiles, sinon impossibles, et ne permettent guère, à part 1a mise en évidence de quelques grandes orientations, de connaître d'une façon plus précise l'évolution de la croissance relative chez l'Agneau.

D'autre part, dans les très nombreux travaux qui y ont été consacrés, les variations de la composition corporelle ont été le plus souvent étudiées non pas en ellesmêmes, mais pour déterminer l'influence de divers facteurs : type d'alimentation, environnement, castration, croisements, et en particulier niveaux de nutrition différents imposant aux animaux des courbes et des vitesses de croissance différentes. Or on sait, précisément par ces travaux, que le développement relatif de divers tissus, régions ou organes peut varier dans une certaine mesure, pour un même animal, selon les conditions d'élevage et de croissance.

Enfin PaLsson (I955), comparant ses travaux à ceux de WALKER et MCMEEKAN (1944), avait observé que certaines mensurations, susceptibles de fournir de bons indices de la composition des carcasses à l'intérieur d'une race donnée, étaient pratiquement sans valeur pour des comparaisons entre races, ou réciproquement.

C'est pourquoi, a fin de faciliter l'étude du rôle de certains facteurs, et notamment des facteurs endocriniens, dans la croissance relative de l'Agneau, il nous a paru souhaitable de mieux connaître d'abord, pour un matériel animal bien déterminé, l'allure du développement relatif de ses différentes parties, dans des conditions de croissance définies.

Tel est l'objet de la présente étude, volontairement limitée aux animaux d'un âge compris entre la naissance et 5 mois et demi, et correspondant à une croissance globale aussi régulière et homogène que possible, dans les conditions habituelles de l'élevage. 


\section{MATÉRIEL E'T MÉTHODES}

Les animaux étudiés doivent pouvoir théoriquement être considérés comme représentant des stades successifs de la croissance d'un même individu. Nous avons rendu cette approximation possible en choisissant des animaux de même race, de conformation homogène, élevés de façon identique et ayant suivi une même courbe de croissance.

Le matériel animal utilisé est l'agneau Mérinos d'Arles du domaine du Merle, dont les caractéristiques d'élevage ont été indiquées par PruD'Hon et al (I966).

\section{A. - Croissance pondérale globale}

Un échantillonnage d'animaux seulement d'après des âges croissants ou seulement d'après des poids croissants ne peut pas convenir à une analyse de référence de la croissance relativ l'on ignore quelle a été l'allure de la croissance globale de ces animaux. Divers auteurs ont en effet montré l'influence modificatrice de la vitesse de croissance sur la composition corporelle des animaux. (McMeekan, i940, sur le Porc; Boccard et Duplan, ig6r, sur l'Agneau).

Il a donc été fait le choix d'une courbe de croissance déterminée, ce qui permet d'autre part d'éviter d'éventuelles interférences, sources possibles de confusions, entre allométries de taille et allométries de croissance.

a) Choix de la courbe de croissance.

A cet effet ont été utilisés les contrôles de croissance effectués lors de deux agnelages précédents et portant chaque fois sur plus de 200 mâles et 200 femelles nés simples. Les données recueillies ont fait ressortir, pour l'ensemble des deux sexes réunis, un poids moyen de naissance très proche de $4 \mathrm{~kg}$ et une vitesse moyenne de gain de $\mathrm{I} 84 \mathrm{~g}$ par jour entre la naissance et 90 jours.

TABLEAU I

Données utilisées pour la courbe de référence

\begin{tabular}{c|c|c|c|c|c}
\hline Période (jours) & $0-10$ & $10-30$ & $30-90$ & $90-130$ & $130-160$ \\
\hline Gain moyen (g) & 220 & 210 & 170 & 140 & 100 \\
\hline
\end{tabular}

D'après ces résultats ont été établies les données prévisionnelles du tableau r, utilisées pour l'établissement de la courbe de référence (fig. I). Cette dernière, représentative par anticipation de la croissance moyenne de l'ensemble des agneaux nés simples du troupeau, s'est révélée très proche de la réalité, puisque la vitesse moyenne de croissance, après contrôle, s'est établie à $186 \mathrm{~g}$ par jour de la naissance à 90 jours.

En fait, les agneaux mâles ont un poids de naissance et une vitesse de croissance un peu supérieurs à ceux des femelles, et la courbe de référence choisie se situe à peu près à égale distance des courbes moyennes de croissance contrôlées des mâles et des femelles (fig. I). C'est volontairement qu'on a choisi une croissance identique pour les mâles et les femelles étudiés, afin d'éliminer l'effet d'une différence de vitesse de gain et de pouvoir analyser l'effet du sexe chez des agneaux ayant suivi une même courbe de croissance globale.

Comme le montre la figure $I$, ceci est assez aisé à obtenir au moins jusque vers Ioo jours ; c'est plus difficile ensuite, où les différences sexuelles s'accusent, ce qui explique les écarts plus importants, par rapport à la courbe de référence, du dernier stade étudié, ainsi que la limitation de l'étude à ce stade ( 160 jours).

L'analyse portera donc sur l'évolution de la croissance relative chez des animaux qui, soit mâles soit femelles et nés simples, auront suivi la courbe de croissance moyenne de référence.

\section{b) Choix des animaux et des stades d'abattage.}

Les observations préalables ont montré qu'un certain nombre d'animaux suivent de manière asse $z$ fidèle cette courbe de croissance moyenne. Beaucoup d'entre eux ont d'ailleurs à la naissance un poids voisin du poids moyen de $4 \mathrm{~kg}$. C'est parmi ces derniers qu'ont été choisis les animaux étudiés. 
De la naissance à 160 jours, il a été prévu 7 stades d'abattage destinés à obtenir une succession de "poids vide " (poids vif moins poids des contenus stomacaux, intestinaux et de la vessie) voisins de ceux indiqués dans le tableau 2. Les moyennes des 4 animaux disséqués à chaque stade, indiquées au tableau 2 bis, font ressortir des résultats très voisins de ceux recherchés.

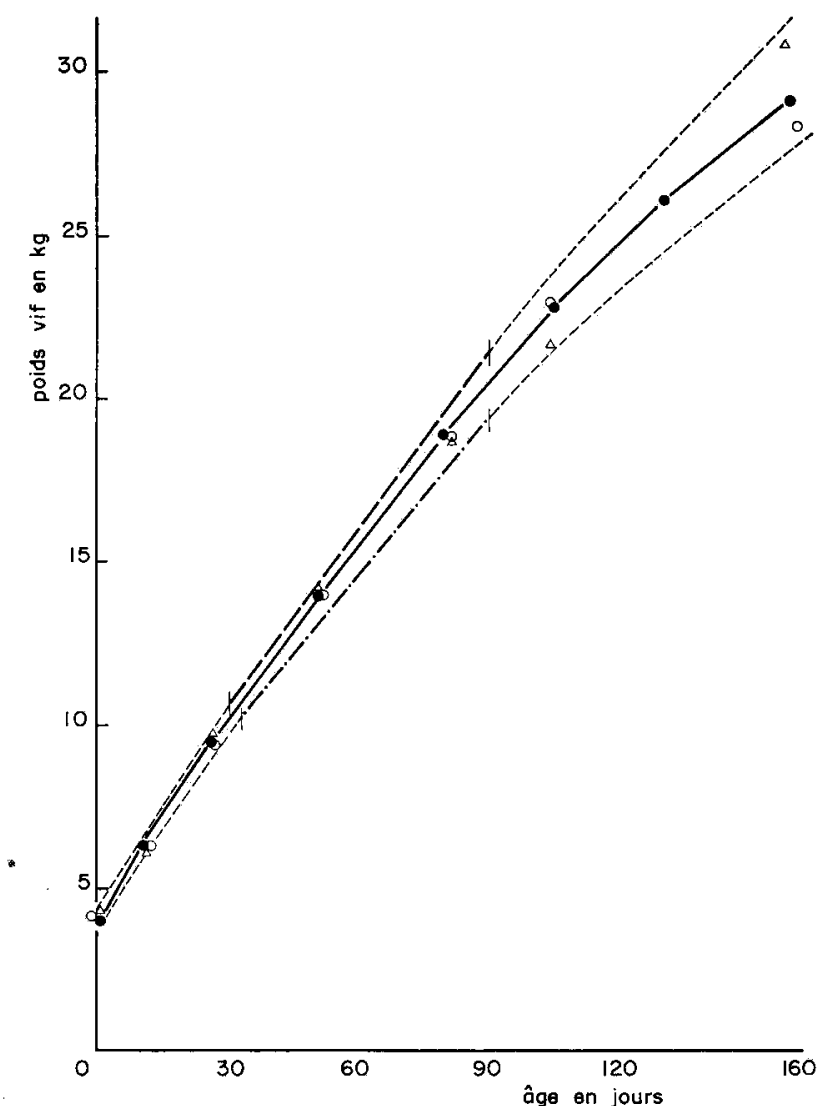

FIG. I. - Courbes de croissance pondèrale et stades d'abattage

-...- Courbe de référence

..... Extrapolations

$$
\text { - - - Croissance moyenne contrôlée }
$$

Animaux abattus $\left\{\begin{array}{lll}\Delta \text { moyenne de } 2 & 0 \\ 0 \text { moyenne de } 2 & \text { o }\end{array}\right.$

Pour chaque stade il a en effet été choisi 2 agneaux mâles et 2 agneaux femelles, nés simples au poids de $4 \mathrm{~kg} \pm 0,2 \mathrm{~kg}$, et dont il a été vérifié par ailleurs qu'ils avaient jusque-là suivi la courbe de croissance moyenne d'une façon suffisamment approchée.

\section{B. - Croissance pondérale relative}

La méthode de dissection complète, préconisée par HammoND (I932) ou ses successeurs (Palsson, I955) a été utilisée selon une technique voisine de celle indiquée par FouRIE (I962).

Les animaux, choisis au sein du troupeau le matin entre 8 et 9 heures, étaient isolés sans nourriture jusqu'à l'abattage prévu à $\mathrm{I}_{4}$ heures et avant lequel ils étaient à nouveau pesés.

Après anesthésie au nembutal-penthotal, et pour éviter de léser certains organes, ils étaient saignés par section de la veine jugulaire et de l'artère carotide préalablement mises à nu, puis pesés de nouveau. La carcasse bouchère était alors préparée et pesée, après séparation de la tête et des extrémités des membres. Reins et capsules surrénales étaient ensuite prélevés et pesés, de 
même que les dépôts de gras interne, selon leur localisation. La dissection musculaire et osseuse et des autres dépôts adipeux a été opérée sur une demi-carcasse, des mesures préalables ayant montré que les régions symétriques ne différaient pas significativement de poids. Cependant pour les régions impaires (vertèbres, sternum, moelle épinière) inégalement séparées par la découpe, leur complément a été récupéré sur l'autre demi-carcasse.

TABLEAU 2

Prévisions pour les stades d'abattage

\begin{tabular}{|c|c|c|c|c|c|c|c|}
\hline Stades & 1 & 2 & 3 & 4 & 5 & 6 & 7 \\
\hline $\begin{array}{l}\text { Age d'abattage } \\
\text { prévu (jours) }\end{array}$ & Naissance & 11 & 25 & 51 & 80 & 105 & 160 \\
\hline $\begin{array}{l}\text { Poids vif } \\
\text { prévu (kg) }\end{array}$ & $4 \pm 0,2$ & 6,400 & 9,400 & $14,000$. & 18,900 & 22,700 & 29,200 \\
\hline $\begin{array}{c}\text { Poids a vide " } \\
\text { prévu (kg) }\end{array}$ & 3,7 à 4 & 6 & 9 & 12 & 15 & 18 & 25 \\
\hline
\end{tabular}

TABLEAU 2 bis

Age et poids d'abattage réels

(moyenne de 4 animaux par stade)

\begin{tabular}{|c|c|c|c|c|c|c|c|}
\hline Stades & 1 & 2 & 3 & 4 & 5 & 6 & 7 \\
\hline $\begin{array}{l}\text { Age d'abattage } \\
\text { (jours) }\end{array}$ & Naissance & 12 & 25 & 51 & 82 & 104 & 160 \\
\hline $\begin{array}{c}\text { Poids vif avant } \\
\text { abattage }(\mathrm{kg})\end{array}$ & 4,130 & 6,200 & 9,650 & 14,120 & 18,720 & 22,330 & 29,670 \\
\hline $\begin{array}{c}\text { Poids " vide " } \\
\text { (kg) }\end{array}$ & 3,926 & 6,001 & 9,127 & 12,401 & 15,511 & 18,706 & 25,324 \\
\hline
\end{tabular}

Tout au long du dépouillement, de l'éviscération et de la dissection, on a cherché à séparer et à peser individuellentent un maximum d'organes. Un matériel précis de pesée a été utilisé, en rapport avec la taille de chaque partie à mesurer (à I mg près de o,2 à roo g ; à o, I g près de Ioo à I $000 \mathrm{~g}$; à $0,5 \mathrm{~g}$ près de $\mathrm{I} \mathrm{kg}$ à $5 \mathrm{~kg}$; à Io $\mathrm{g}$ près de $5 \mathrm{~kg}$ à Io $\mathrm{kg}$ ).

Pour les muscles, disséqués selon une technique décrite antérieurement (BÉnḱvent, I968), seule la partie charnue a été considérée, les régions tendineuses en étant séparées à l'endroit où elles devenaient nettement distinctes, toujours identiquement pour chaque muscle. Les pièces osseuses ont été soigneusement nettoyées, par enlèvement du périoste, section et grattage de tous autres tissus adhérents, sauf cartilagineux. Le tube digestif et la vessie ont été vidés de leur contenu dont la pesée a permis par soustraction d'obtenir le " poids vide".

Les déchets divers (tissu conjonctif, tendons, ligaments, vaisseaux, ganglions, nerfs, débris de grattage des os, sang demeuré dans la cavité thoracique et les gros vaisseaux) ont été récupérés et pesés. Enfin des précautions particulières ont été prises pour limiter au maximum les pertes par évaporation. A cette fin, tous les organes ou régions en cours de dissection, de même que les déchets, ont été maintenus enveloppés de bandes de gaze légèrement humides, jusqu'au moment. de leur manipulation.

Les extrémités (tête et extrémités des membres) encore entourées de leur peau et placées dans des sacs en matière plastique, en chambre froide à $+2^{\circ}$, peuvent demeurer ainsi plusieurs jours sans perdre pratiquement de lẹur poids (moins de I p. Ioo) et être disséquées en dernier lieu. De même les parties non disséquées dans la journée sont d’un jour à l'autre conseryées au 
frigorifique à $+2^{\circ}$, enveloppées dans une gaze légèrement humide, puis dans la peau, le tout enfermé dans un sac en matière plastique.

Toutes les parties et les déchets ayant été chaque fois pesés, on a pu faire une évaluation exacte des pertes, dues essentiellement à l'évaporation.

Les pertes les plus élevées correspondent à la dissection de la tête et des os, qui exigent les manipulations les plus longues. Elles s'établissent entre 3 et 6 p. roo, avec une moyenne d'environ 5 p. IOO.

Par contre, lors du prélèvement des organes et de la dissection musculaire de la demi-carcasse les pertes sont beaucoup moins sensibles : elles se situent entre 0,5 et 2,5 p. Ioo, avec une moyenne de 1,5 p. Ioo.

De sorte que les pertes totales, résultant de l'ensemble des dissections, représentent en moyenne environ 3 p. 100 du poids vif. Ce pourcentage, difficile à améliorer compte tenu du nombre élevé des manipulations et de l'assez longue durée de l'ensemble des opérations, est d'ailleurs d'autant plus acceptable qu'il varie peu d'une dissection à l'autre et n'influence donc que très peu les résultats comparatifs.

\section{C. - Exploitation des données recueillies}

Pour un organe qui en cours de croissance suit la relation d'allométrie $\left(y=k x^{\alpha}\right)$ mise en évidence par HuXLEY (1932), si l'on représente en coordonnées logarithmiques l'évolution de son poids $(y)$ en fonction de celui du corps $(x)$, les points représentatifs sont alignés sur une même droite de pente $\alpha$.

La confrontation des graphiques ainsi obtenus et des différents coefficients d'allométrie $(\alpha)$ permet de comparer l'allure et la vitesse d'évolution relatives des différentes parties d'un organisme.

En raison de sa commodité nous avons utilisé ce mode de représentation pour conduire notre analyse, en supposant (la grande généralité de la relation d'allométrie étant maintenant admise) que la plupart des organes devaient obéir, durant des périodes à déterminer, à une telle relation.

Toutefois, en raison même des nécessités imposées par notre travail, il existe un écueil à l'utilisation de cette méthode, qui nous oblige à en préciser quelque peu les conditions d'emploi.

En effet, les mêmes animaux ne peuvent être suivis au long de la période đe croissance étudiée (comme cela pourrait se faire pour des mensurations corporelles) puisqu'il y a nécessité d'abattage à des stades successifs.

\section{TABLEAU 3}

Variabilité pondérale de divers organes pour quatre animaux de mếme poids vide (I $2400 \mathrm{~g}$ )

\begin{tabular}{|c|c|c|c|c|c|c|c|c|}
\hline & & & Coeur & Cerveau & Estomac & $\begin{array}{c}\text { Somme } \\
\text { des } \\
\text { muscles }\end{array}$ & Thymus & Sang \\
\hline \multirow{3}{*}{$\begin{array}{c}\text { Poids } \\
\text { en } \\
\text { grammes }\end{array}$} & \multicolumn{2}{|c|}{ Moyenne } & 74,3 & 97,5 & 269 & 4503 & 80,4 & 825 \\
\hline & \multirow{2}{*}{$\begin{array}{l}\text { Valeurs } \\
\text { extrêmes }\end{array}$} & Minim. & 67,5 & 95,2 & 258 & 4352 & 63,7 & 780 \\
\hline & & Maxim. & 89,7 & 98,6 & 284 & 4616 & 97,9 & 900 \\
\hline \multicolumn{3}{|c|}{$\begin{array}{c}\text { Coefficient de variation } \\
\text { en } \%\end{array}$} & 14 & 1,6 & 4,6 & 2,4 & 19,9 & 6,4 \\
\hline
\end{tabular}

Les organes étudiés appartiennent donc à des animaux différents, et malgré les précautions observées pour le choix de ces derniers, il subsiste entre eux à chaque stade une certaine variabilité, plus ou moins importante selon les organes. Le tableau 3 en donne une illustration; il concerne les 4 animaux du stade 4 qui ont chacun (à quelques grammes près) un poids vide très voisin de $12400 \mathrm{~g}$ (les organes choisis ne sont pas - tout au moins à ce stade — influencés par le sexe).

Cette variabilité donne au tracé par points individuels des irrégularités qui risquent de masquer la véritable relation d'allométrie ou ses changements éventuels (fig. 3 A ; fig. 4 A). Il serait 
donc souhaitable de pouvoir utiliser en chaque point, pour éliminer les effets de la variabilité, les moyennes d'un nombre assez élevé d'animaux chez lesquels on pourrait supposer que les poids de chaque organe sont normalement distribués. Malheureusement la méthode de dissection complète d'un animal comme le Mouton en limite évidemment 1'usage à un nombre restreint à chaque stade.

Cependant, les pertes subies par les organes variant au plus de 2 p. roo entre deux dissections, la précision des pesées étant celle qui a été indiquée, et le mode opératoire de dissection étant toujours suivi identiquement par les mêmes manipulateurs, il s'ensuit que la marge de variation pondérale enregistrée pour un organe entre deux animaux de développement voisin est pour la plus grande part imputable à la variabilité individuelle. Il est difficile de le démontrer strictement mais une vérification indirecte peut en être faite en suivant graphiquement l'évolution d'organes très semblables (comme 2 phalanges successives) subissant chez l'animal des variations identiques et de même sens. Si les variations observées sont bien dues essentiellement à la variabilité individuelle, les tracés par points individuels doivent, pour ces organes variant identiquement, être sensiblement parallèles et offrir les mêmes irrégularités. C'est bien ce que l'on peut constater sur la figure 2, relative aux première et deuxième phalanges externes du pied antérieur et du pied postérieur (animaux mâles).

Dans de telles conditions les moyennes de 2 animaux seulement peuvent déjà amortir suffisamment la variabilité pour donner des tracés assez réguliers, permettant de suivre au moins les grandes tendances évolutives de la croissance relative. Cela est montré notamment par le parallélisme fréquent obtenu dans ces conditions entre les tracés des mâles et ceux des femelles pour un même organe (fig. 3 : muscles sus-épineux; os de la tête ; tube digestif). Les changements observés, qui se répètent fidèlement mais avec un certain décalage d'un sexe à l'autre, ne peuvent être dus au hasard mais traduisent évidemment une évolution comparable de la croissance relative.

C'est pourquoi nous avons fréquemment utilisé cette méthode graphique d'appréciation, particulièrement pour une étude descriptive des phénomènes, le tracé par points successifs étant par ailleurs assez suggestif et permettant notamment des comparaisons commodes.

Des moyennes de 4 animaux par stade ont parfois été utilisées, lorsque les organes ont une évolution identique, très proche dans chaque sexe. Le tracé est alors très voisin de ce que donneraient les droites de régression calculées (fig. 4 : cœur ; cerveau).

Cependant, lorsque la variabilité de l'organe est grande ou que les changements de pente observés sont faibles ou douteux, l'appréciation visuelle devient plus imprécise ou ne permet pas de lever certaines indéterminations. Des méthodes de calcul statistique peuvent alors être utilisées, et la droite de régression calculée peut fournir une estimation valable de la droite et du coefficient d'allométrie. Dans une étude analogue effectuée sur le Lapin, CANTIER et al (1969) justifient d'ailleurs la détermination des coefficients d'allométrie par le calcul des coefficients de régression de $\log y$ sur $\log x$ ( $y$ et $x$ représentant respectivement le poids de l'organe étudié et le a poids vide * du corps).

En cas de doute sur l'existence d'un changement de pente ou sur le stade auquel il se produit, ces mêmes auteurs s'efforcent, à l'aide du calcul, de lever cette indétermination d'une manière objective. A cet effet, ils testent systématiquement dans chaque cas une vingtaine d'hypothèses leur paraissant graphiquement plausibles. Ils retiennent comme le plus vraisemblable l'ajustement à la droite ou aux deux ou trois droites successives de régression qui correspond à la plus faible variance résiduelle (variance des écarts à la ou aux droites de régression).

Nous avons opéré d'une manière analogue. Cependant, compte tenu des observations faites plus haut sur les bonnes indications données par nos graphiques en moyennes de 2 ou de 4 animaux, nous avons utilisé plus largement la méthode graphique pour nous suggérer les hypothèses à soumettre au calcul, afin de réduire ces dernières à 2 ou 3 dans chaque cas, les moins douteuses nous semblant suffisamment apparentes graphiquement. Les deux méthodes ne s'excluent évidemment pas, mais se complètent.

Les stades auxquels se situent les changements d'allométrie ou les zones à l'intérieur desquelles se placent le plus grand nombre de changements correspondent à des " poids vide " précis qui résultent du choix même de nos stades. Mais il est évident qu'une certaine variabilité existe autour de ces stades, que nous n'avons pu apprécier en raison du nombre trop réduit d'animaux. Il ne faut donc les prendre que comme des repères provisoires, déterminant de façon approximative les périodes où se situent les principaux changements observés, pour la race étudiée et dans les conditions de croissance indiquées. 


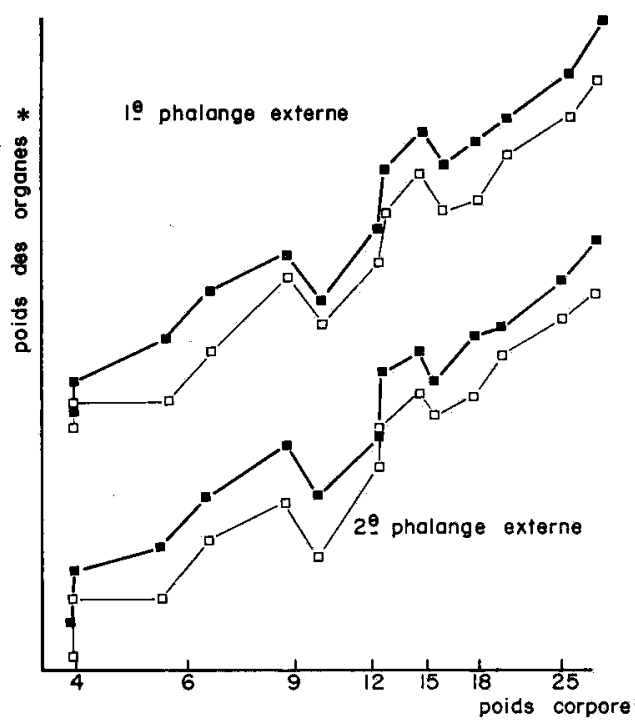

FIG. 2.

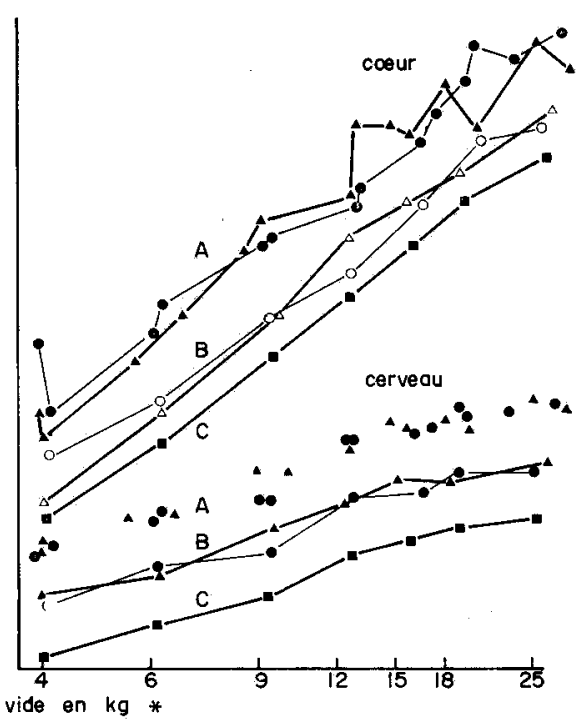

Fig. 4.

FIG. 2. - Évolutions parallèles d'organes semblables

(mâles : tracés par points individuels)

- membre antérieur

* Échelle logarithmique

$\square-\square$ membre postérieur

FIG. 4. - Atténuation de la variabilité

$\mathrm{A}=$ points individuels

$\mathrm{B}=$ moyenne de 2

$\triangle \Delta$ mâles

o femelles

* Échelle logarithmique

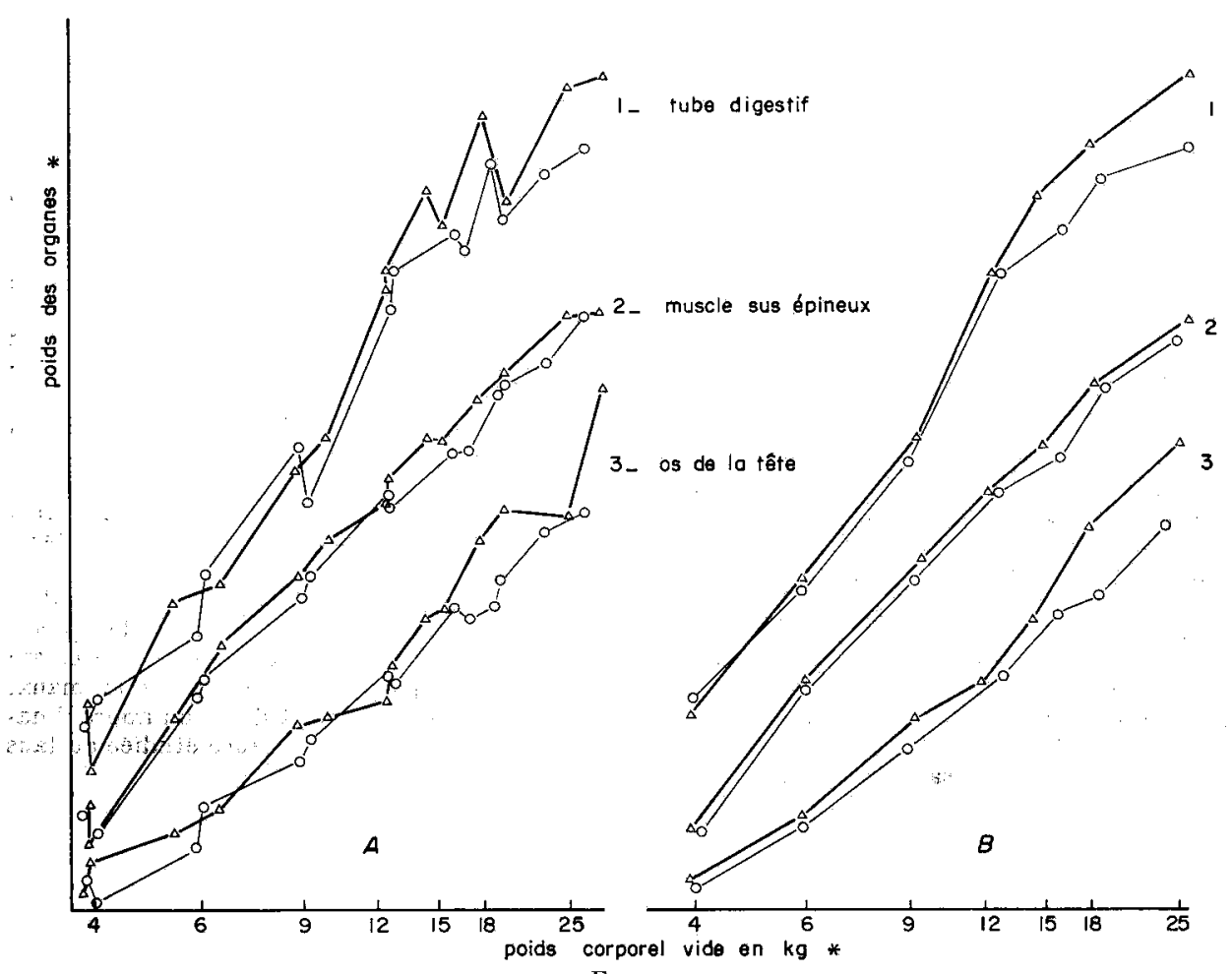

FIG. 3.

FIG. 3. - Atténuation graphique de la variabilité

$A=$ points individuels $\quad \Delta \longrightarrow \Delta$ mâles

$\mathrm{B}=$ moyenne de deux animaux 0 -o femelles 


\section{RÉSULTATS}

\section{I. - Premiers stades postnataux (1-3)}

Jusqu'aux environs de $9 \mathrm{~kg}$ de poids vide (stade 3,25 jours) les principaux tissus et organes évoluent à des vitesses différentes mais de façon très proche chez les mâles et les femelles, et sans subir de variations apparentes importantes de leur coefficients d'allométrie. C'est pourquoi nous avons calculé ces coefficients d'allométrie pour les trois premiers stades, en considérant l'ensemble des mâles et des femelles sans distinction de sexe, ceci par l'intermédiaire des droites de régression qui correspondent à l'équation générale :

$$
\log y=\alpha \log x+\log b
$$

$x$ représentant le poids vide du corps, et $y$ le poids correspondant de l'organe étudié.

Les résultats qui seront discutés plus loin, sont rassemblés dans le tableau 4, et sont reportés graphiquement à la figure 5 .

\section{TABLEAU 4}

Premiers stades postnataux: parametres ( $\alpha$ et $b$ ) des droites d'allométrie $(\log y=\alpha \log x+\log b)$

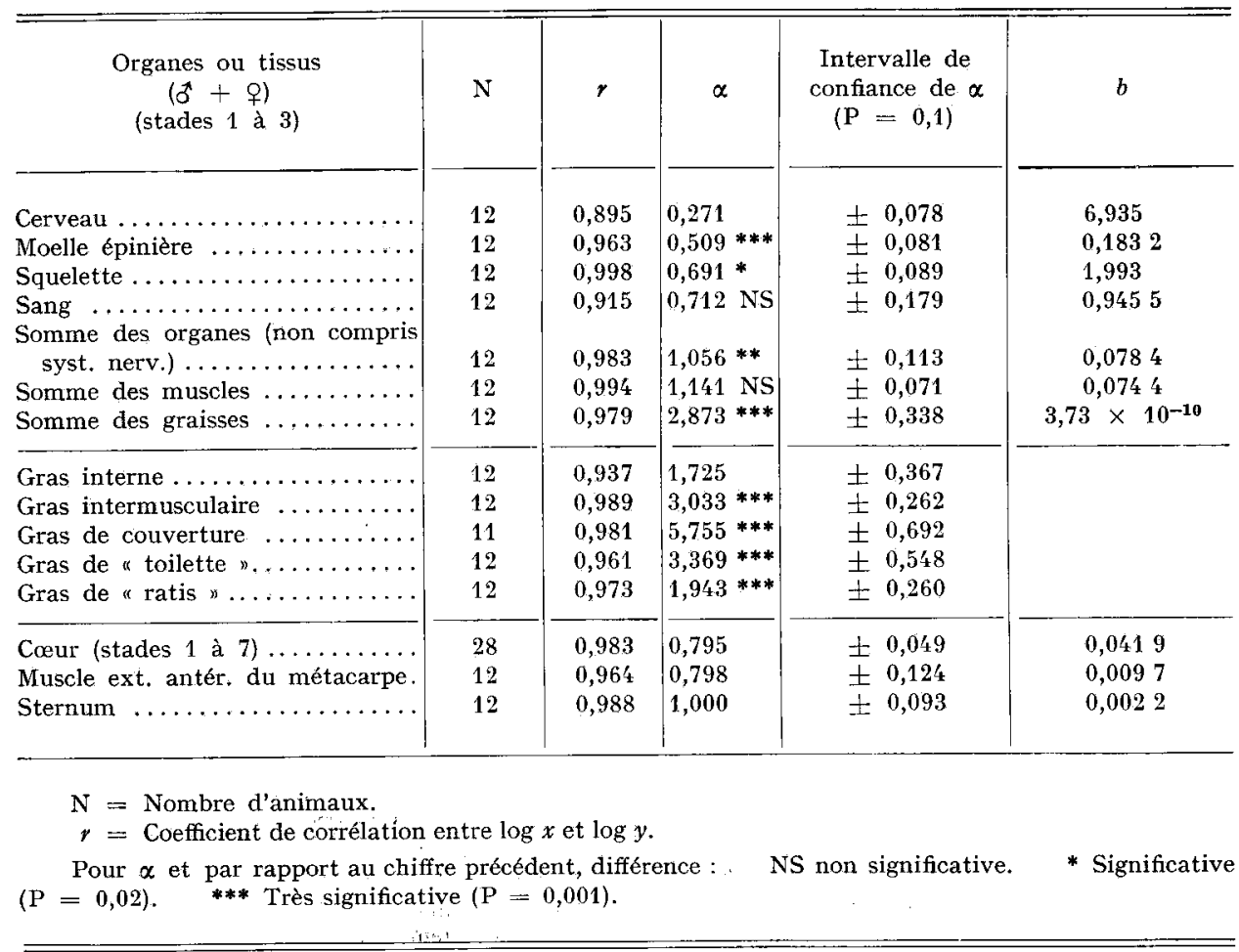




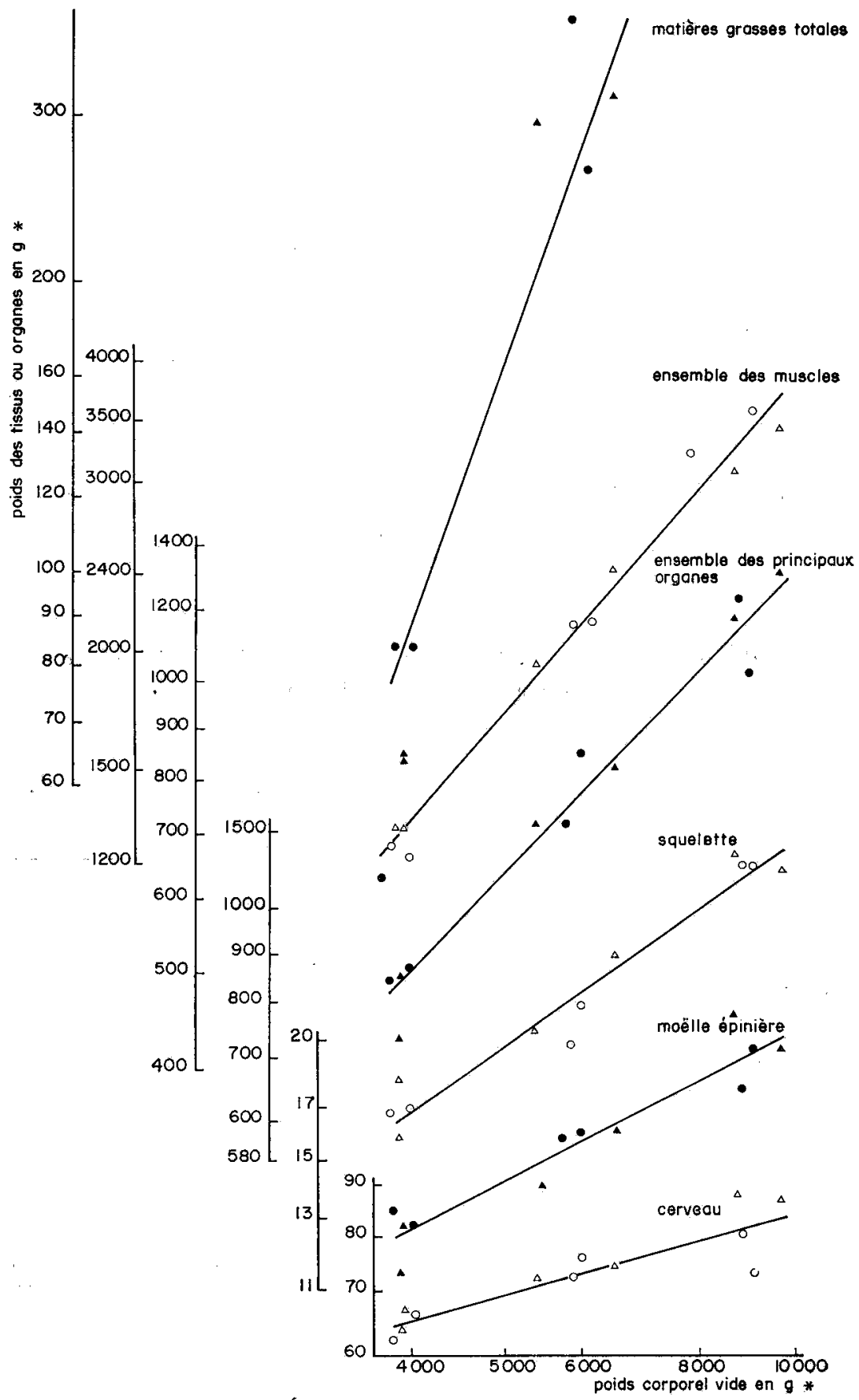

Fig. 5. - Évolution des principaux tissus et organes lors des premiers stades post-nataux

* Échelle logarithmique $\quad \Delta_{\Delta}$ mâles

o femelles 


\section{2. - Inégalités de développemeni selon le sexe}

La méthode graphique, utilisée dans l'esprit qui a été précisé plus haut, permet d'apprécier d'un coup d'œil, sur l'ensemble de la période étudiée, un certain nombre d'analogies ou de différences suivant le sexe, quant au développement relatif des différentes parties. Cette appréciation étant surtout descriptive, nous ne relèverons ici que les faits les plus nettement apparents graphiquement, et nous les commenterons au cours de la discussion.

\section{a) Analogies.}

Certains tissus ou organes semblent évoluer de façon semblable chez les mâles et les femelles, les tracés graphiques étant voisins et se recoupant mutuellement. Ainsi en est-il pour l'ensemble des muscles du squelette, le sang, la moelle épinière (fig. 6 a), le cour, le cerveau (fig. 4).

\section{b) Différences.}

Malgré l'analogie d'évolution de l'ensemble du système musculaire, il peut exister des différences locales plus ou moins nettes : les muscles du cou sont toujours, à tous les stades, plus importants chez les mâles ; il en est de même du muscle sus-épineux, alors que la masse commune du dos paraît être, dans les premiers stades, légèrement plus lourde chez les femelles (fig. 6 b).

Mais les différences les plus apparentes concernent le squelette, certains organes particuliers ainsi que la somme des organes internes, et surtout le tissu gras.

- A poids vide et âge égaux, l'ensemble du squelette est plus lourd chez les mâles, la différence s'accusant surtout après 9 à ro $\mathrm{kg}$ de poids vide (fig. $6 \mathrm{c}$ ). Il en est ainsi pour la plupart des régions sauf pour la cage thoracique, dont l'évolution est d'ailleurs la plus rapide du système osseux, et pour laquelle les points représentatifs sont très proches chez les deux sexes, avec cependant une légère différence en faveur des mâles après $9 \mathrm{~kg}$. La différence la plus nette provient des os de la tête constamment plus lourds chez les mâles, l'écart s'accentuant largement après 15 à I6 kg de poids vide (stade $5 ; 80$ jours) en raison probablement du développement progressif des cornes chez le mâle (fig. 3).

En ce qui concerne les organes, c'est le foie qui montre la plus précoce différence, devenant plus important chez les mâles dès le poids vide de $6 \mathrm{~kg}$ (fig. $6 d$ ). De même les reins sont plus lourds chez le mâle aux stades précoces, mais à partir de $15 \mathrm{~kg}$ de poids vide la différence n'est plus sensible et semble même s'inverser (fig. $6 d$ ).

Le tube digestif, tant dans sa totalité (fig. 3 B) que dans ses parties (estomacs fig. $6 c$; intestins fig. $6 d$ ) voit son développement s'accentuer davantage chez les mâles à partir de I $2 \mathrm{~kg}$ de poids vide (stade $4 ; 50$ jours). De même fait la rate, malgré sa forte variabilité (fig. $6 \mathrm{~d}$ ).

Quant aux gonades, elles montrent une évolution tout à fait dissemblable. L'ovaire, au développement relatif plus rapide que celui du testicule jusque vers I2 $\mathrm{kg}$ de poids vide, montre par la suite une certaine régression, ou au moins - compte tenu de la forte variabilité possible - une stagnation jusqu'au dernier stade de la période analysée. Le testicule au contraire subit, dès le stade de $15,5 \mathrm{~kg}$ de poids vide (stade $5 ; 80$ jours) un développement explosif (fig. 7). 


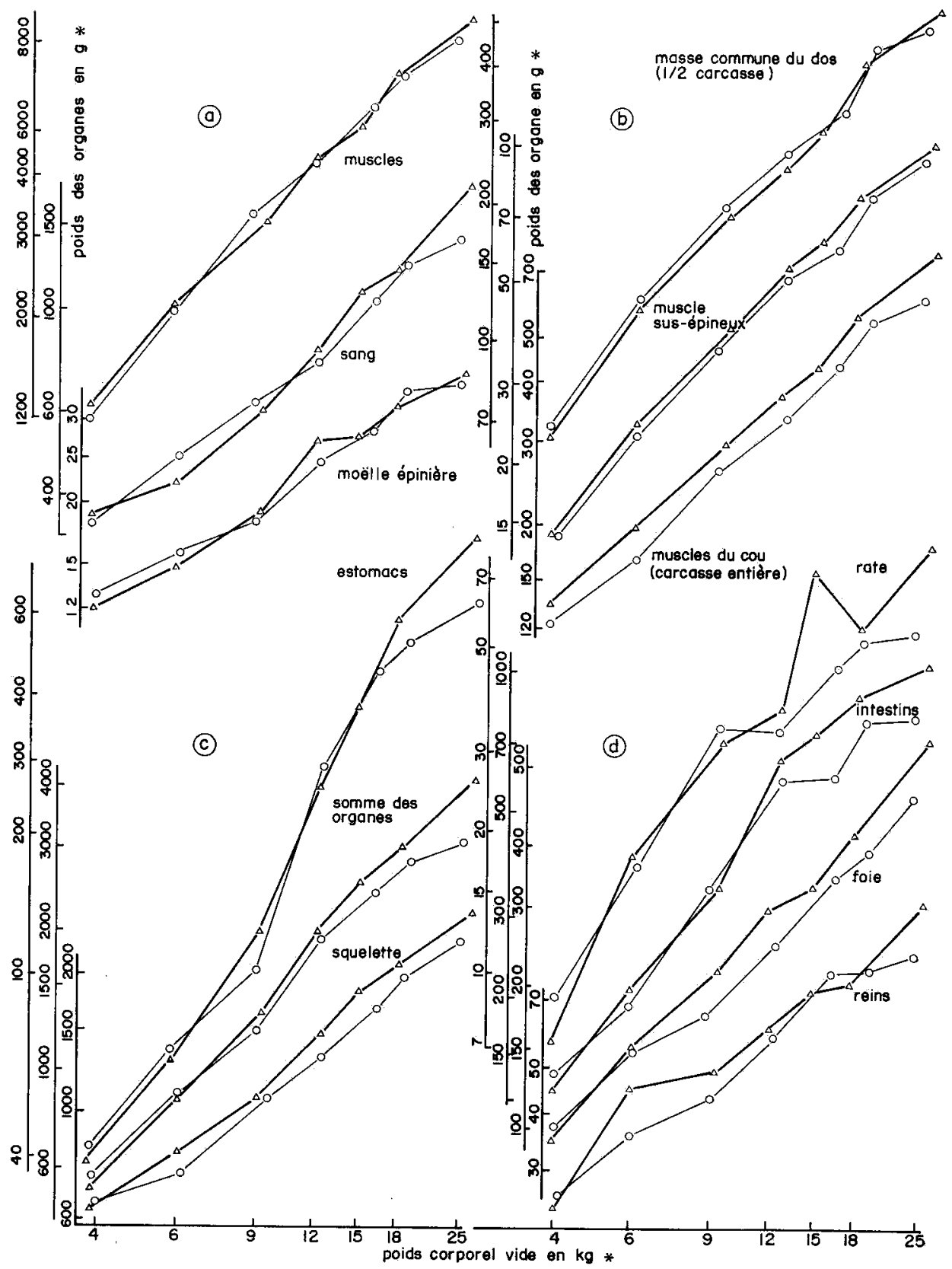

Fig. 6. - Évolutions analogues ou différentes, selon le sexe, de divers tissus ou organes

$\stackrel{\Delta \longrightarrow \text { mâles }}{\triangle \longrightarrow}\{$ femelles (moyenne de 2 animaux) *Échelle logarithmique 
La somme des muscles étant analogue chez le mâle et la femelle, malgré certaines différences locales signalées plus haut, nous avons également représenté l'évolution de la somme des principaux organes (tube digestif; appareil uro-génital (et mamelle chez les femelles) ; trachée, cour, poumons ; foie, rate, reins ; système nerveux ; glandes endocrines). Cette somme des organes montre, après le poids vide de $9 \mathrm{~kg}$, un écart grandissant en faveur des mâles, bien que les deux courbes aient une allure comparable (fig. $6 \mathrm{c}$ ). Le squelette demeurant par ailleurs plus lourd chez le mâle, on peut donc s'attendre à ce que le tissu gras prenne plus d'importance chez la femelle, à partir de $9 \mathrm{~kg}$ de poids vide.

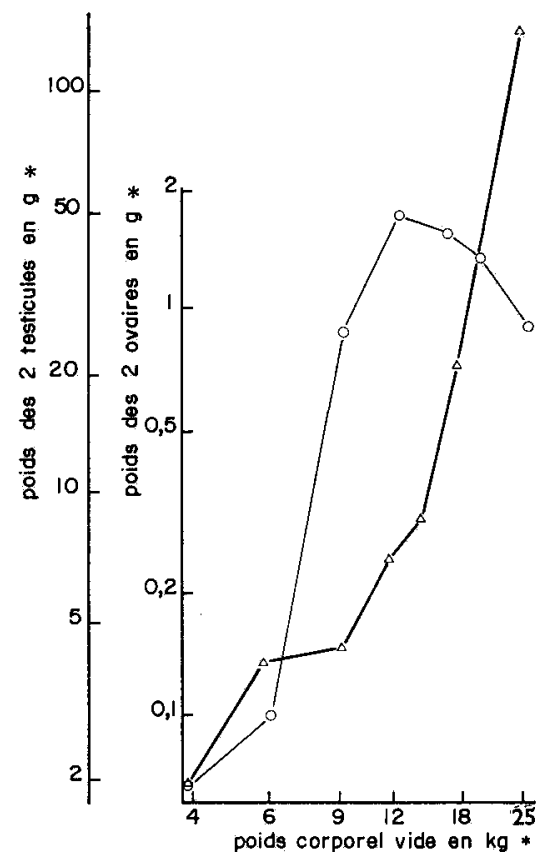

FIG. 7. - Evolutions dissemblables des gonades

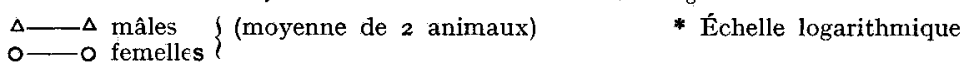

- L'évolution du tissu gras est en effet particulièrement intéressante à comparer chez les deux sexes. Considéré dans sa totalité comme dans ses principaux lieux de dépôt, son développement est toujours plus important chez les femelles (fig. $8 a$ et et $8 \mathrm{~b}$ ). Cependant jusqu'au poids vide de $9 \mathrm{~kg}$ (stade $3 ; 25$ jours) l'écart entre les deux sexes demeure faible ou très faible, et les courbes représentatives demeurent, même dans leurs changements de pente, remarquablement parallèles. Jusque-là on note aussi une légère tendance du coefficient d'allométrie, très élevé au départ, à diminuer dès le stade 2 (6 kg de poids vide) sauf pour le gras intermusculaire.

Mais c'est surtout à partir du stade 3 que la différence entre les sexes s'accuse de manière brutale à cet égard. Alors que chez les femelles le tissu gras, bien que ralentissant son développement, continue à croître rapidement dans toutes les régions, il se produit chez les mâles un très foṛt ralentissement (gras intermusculaire) ou une stagnation (gras total) ou même une forte régression (gras interne; gras de 
couverture) (fig. $8 a$ et $8 b$ ). Seuls présentent une exception relative les dépôts adipeux de la " toilette " et du " ratis " (le premier de ces termes du langage courant désigne la portion du péritoine enveloppant des estomacs; le "ratis " correspond aux portions mésentériques enveloppant les intestins).

Le gras du " ratis " continue à se développer de façon parallèle chez les deux sexes, bien qu'avec une différence de plus en plus accusée en faveur des femelles; il en est de même pour la " toilette ", mais avec des écarts encore plus marqués (fig. 8 b).

Ainsi les mâles subissənt un véritable amaigrissement passager entre $9 \mathrm{~kg}$ et I $5 \mathrm{~kg}$ de poids vide (stade $3 ; 25$ jours à stade $5 ; 80$ jours). Puis le tissu gras y reprend son développement de façon de nouveau sensiblement parallèle à ce qu'il est chez les femelles, voire même un peu plus rapide, mais un certain décalage très apparent est maintenu.

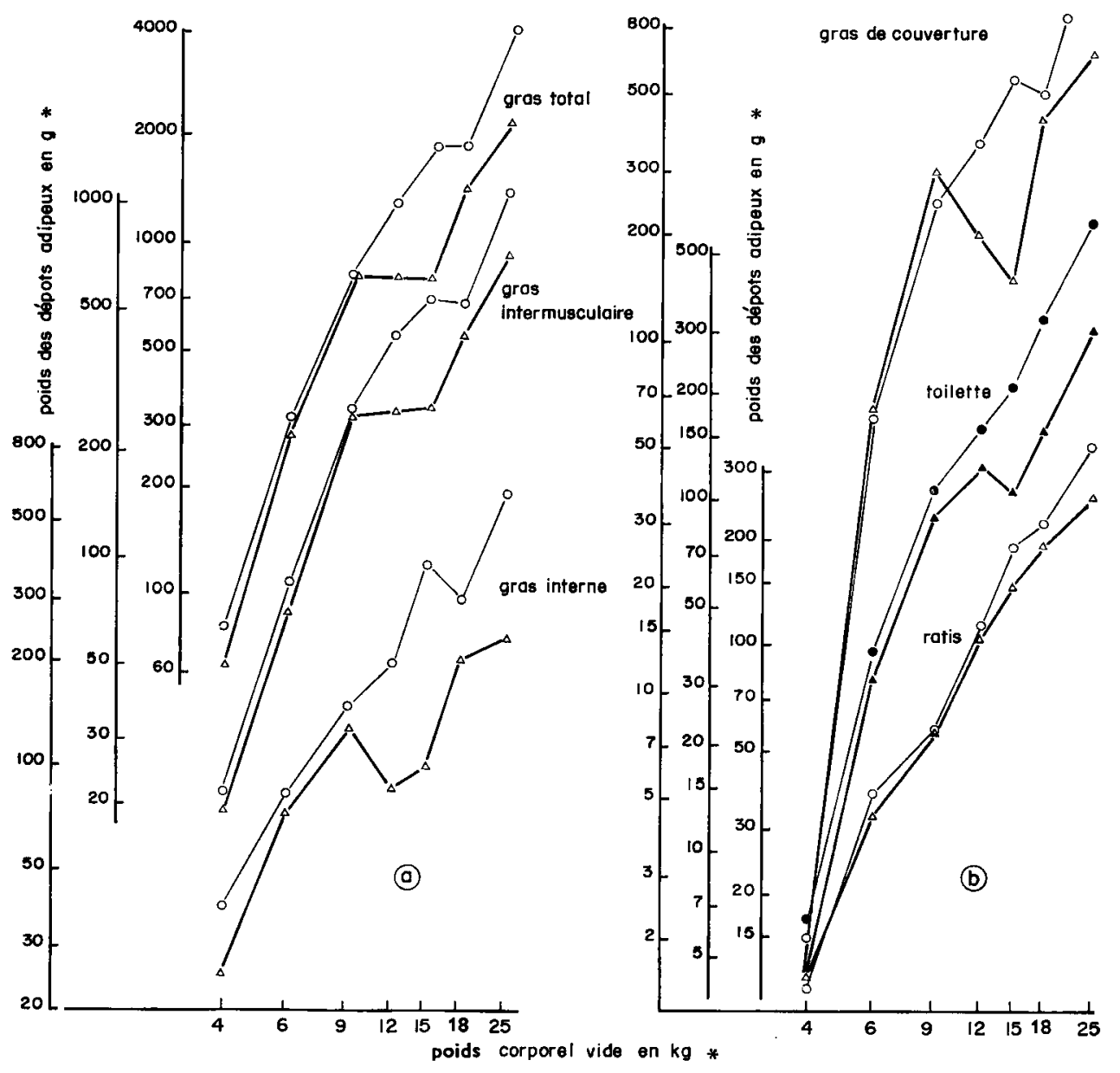

FIG. $8 a$. et 8 b.- Évolutions différentes des tissus adipeux selon le sexe et les lieux de dépót

$\Delta \longrightarrow \Delta$ mâles
$0 \longrightarrow 0$ femelles (moyenne de 2 animaux)

* Échelle logarithmique 
Sur le vu des graphiques, les différences entre les deux sexes se situent dans l'ordre d'importance décroissant suivant : gras interne, gras de couverture, gras intermusculaire, " toilette ", " ratis ".

\section{3. - Evolution de la croissance relative des différentes parties de l'organisme}

a) Évolution à variation continue.

Un certain nombre de tissus ou d'organes semblent montrer une variation continue du coefficient d'allométrie $(\alpha)$ d'un stade à l'autre. Il s'agit tantôt d'une décroissance régulière, la succession des droites joignant un stade au suivant donnant alors l'apparence générale d'une courbe régulière à concavité tournée vers le bas (tissu gras total, gras intermusculaire, "toilette ", chez les femelles entre les stades I à 5, fig. $8 a$ et $8 b$ ) ; tantôt au contraire se présente un accroissement régulier de $\alpha$, donnant au tracé l'apparence d'une courbe à concavité tournée vers le haut (peau, surtout chez les femelles, fig. 9) ; tantôt enfin le tracé prend l'apparence d'une courbe sigmoïde (maxillaire inférieur ; muscles de la tête ; estomacs, fig. 9).

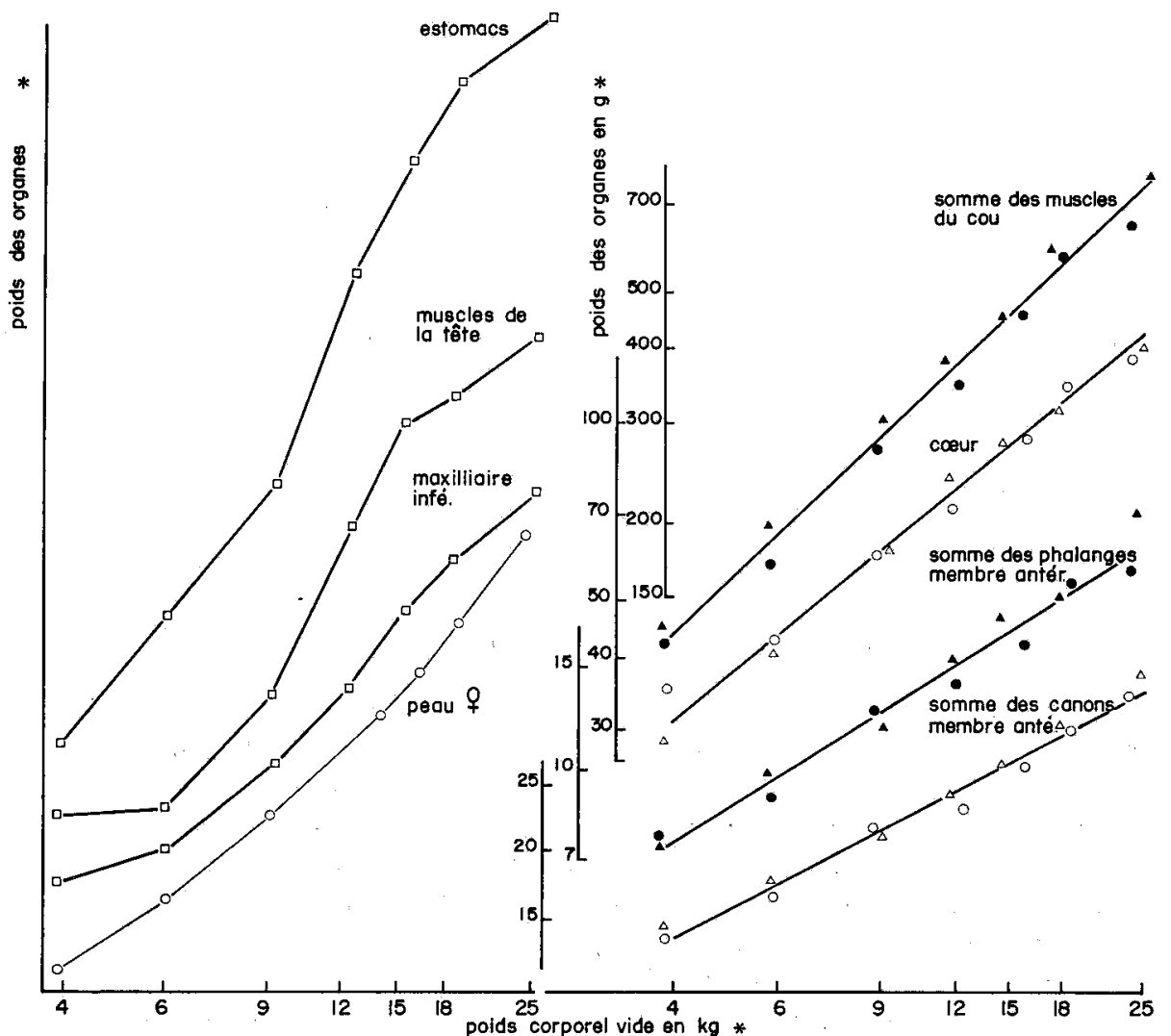

FIG. 9. - Évolution à variation continue

FIG. Io. - Evolution de divers organes selon de certains organes

une seule droite d'allométrie

$\square \longrightarrow \square$ moyenne de 4 animaux * Échelle logarithmique $\Delta_{\Delta}$ mâles $\{$ (moyenne de 2 animaux)

0 - 0 femelles 4 o femelles $\{*$ Échelle logarithmique 
Donner dans ces différents cas une série de valeurs successives pour le coefficient d'allométrie serait sans grande signification, d'une part parce que le nombre d'animaux à chaque stade ne serait pas assez élevé pour assurer un fondement solide au calcul, d'autre part parce que de tels organes n'obéissent peut-être pas à la relation simple classique d'allométrie.

b) Évolution sans variation importante.

A l'opposé de ces tendances, d'autres régions corporelles montrent par rapport à celui du poids vide un taux d'accroissement à peu près constant et analogue dans les deux sexes, se traduisant par un coefficient d'allométrie sans variations apparentes importantes tout au long de la période étudiée, et donc par l'existence d'une seule droite d'allométrie.

Dans ce cas, c'est d'ailleurs l'ajustement à une seule droite de régression (stades I à 7) qui laisse la variance résiduelle la plus faible. Il en est ainsi par exemple pour le cour, les muscles du cou, les os canon, les phalanges (tabl. 5 ; fig. Io).

TABLEAU 5

Ajustement à une seule droite d'allométrie

\begin{tabular}{|c|c|c|c|c|c|c|}
\hline $\begin{array}{c}\text { Organes } \\
(\hat{0}+\text { }+ \text { ) } \\
\text { ( stades } 1 \text { à } 7)\end{array}$ & $\mathrm{N}$ & $r$ & $\alpha$ & $\begin{array}{c}\text { Intervalle } \\
\text { de confiance } \\
\operatorname{de} \alpha(P=0,1)\end{array}$ & $\begin{array}{l}\text { Variance } \\
\text { résiduelle }\end{array}$ & $b$ \\
\hline Cour ......... & 28 & 0,983 & 0,795 & $\pm 0,049$ & 0,001575 & 0,0419 \\
\hline Muscles du cou ..... & 27 & 0,986 & 0,930 & $\pm 0,058$ & 0,001699 & 0,0566 \\
\hline Os canons antér. ... & 28 & 0,985 & 0,527 & $\pm 0,030$ & 0,000630 & 0,1737 \\
\hline Phalanges antér. .... & 28 & 0,974 & 0,639 & $\pm 0,049$ & 0,001630 & 0,0365 \\
\hline
\end{tabular}

$\mathrm{N}=$ Nombre d'animaux.

$r=$ Coefficient de corrélation entre $\log x$ et $\log y$.

c) Changements d'allométrie et translations ou décalages.

Cependant de nombreux organes ou tissus dont le coefficient d'allométrie ne semble ni varier de façon continue ni demeurer constant tout au long de la période étudiée, présentent en un point particulier (ou parfois deux) un changement de pente de la droite d'allométrie, ou un véritable décalage de celle-ci.

Certains de ces changements sont suffisamment nets graphiquement pour qu'il ne puisse y avoir de doute à leur sujet (estomacs ; intestins ; somme des organes ; matières grasses : fig. $6 c, 6 d$ et $8 a$; thymus, fig. II).

Pour d'autres on peut douter de leur existence ou de leur emplacement, le simple relevé graphique permettant d'hésiter entre deux ou trois possibilités apparentes (une seule droite d'allométrie, ou deux droites avec indécision quant au stade de changement de pente éventuel).

Dans la plupart de ces cas, le tracé par points successifs montre entre les deux 
sexes soit une évolution identique, soit - lorsqu'il y a une différence - une évolution parallèle, les changements de pente apparents se plaçant aux mêmes endroits. C'est pourquoi, dans le but de déterminer les hypothèses statistiquement les plus probables, nous avons dans ces cas regroupé les données de l'ensemble des deux sexes, afin

TABLEAU 6

Ajustement à deux droites d'allométrie (ou à une seule)

\begin{tabular}{|c|c|c|c|c|c|c|c|}
\hline $\begin{array}{c}\text { Tissus ou organes } \\
\left(\delta^{*}+\varnothing\right)\end{array}$ & $\begin{array}{l}\text { Stades } \\
\text { (droites de } \\
\text { régression) }\end{array}$ & $N$ & $r$ & $\alpha$ & $\begin{array}{c}\text { Intervalle de } \\
\text { confiance de } \alpha \\
(\mathrm{P}=0,1)\end{array}$ & $\begin{array}{l}\text { Variance } \\
\text { résiduelle }\end{array}$ & $b$ \\
\hline Squelette & $\begin{array}{l}1-3 \\
4-7\end{array}$ & $\begin{array}{l}12 \\
16\end{array}$ & $\begin{array}{l}0,998 \\
0,911\end{array}$ & $\begin{array}{l}0,691 \\
0,775 \mathrm{NS}\end{array}$ & $\begin{array}{l} \pm \quad 0,089 \\
\pm \quad 0,052\end{array}$ & 0,001382 & $\begin{array}{l}1,993 \\
0,005\end{array}$ \\
\hline Sang & $\begin{array}{l}1-3 \\
4-7\end{array}$ & $\begin{array}{l}1.2 \\
16\end{array}$ & $\begin{array}{l}0,915 \\
0,897\end{array}$ & $\begin{array}{l}0,712 \\
0,992 \mathrm{NS}\end{array}$ & $\begin{array}{l} \pm 0,179 \\
\pm 0,213\end{array}$ & 0,002970 & $\begin{array}{l}0,945 \\
0,150\end{array}$ \\
\hline Muscles & $\begin{array}{l}1-2 \\
3-7\end{array}$ & $\begin{array}{r}8 \\
20\end{array}$ & $\begin{array}{l}0,982 \\
0,972\end{array}$ & $\begin{array}{l}1,213 \\
0,954 * *\end{array}$ & $\begin{array}{l} \pm 0,058 \\
\pm 0,098\end{array}$ & 0,000258 & $\begin{array}{l}0,055 \\
0,569\end{array}$ \\
\hline $\begin{array}{l}\text { Somme des organes } \\
\text { (syst. nerv. compris) }\end{array}$ & $\begin{array}{l}1-3 \\
4-7\end{array}$ & $\begin{array}{l}12 \\
16\end{array}$ & $\begin{array}{l}0,984 \\
0,902\end{array}$ & $\begin{array}{l}0,979 \\
0,830 \mathrm{NS}\end{array}$ & $\begin{array}{l} \pm 0,100 \\
\pm 0,186\end{array}$ & 0,001780 & $\begin{array}{l}0,172 \\
0,827\end{array}$ \\
\hline Cerveau & $\begin{array}{l}1-3 \\
4-7\end{array}$ & $\begin{array}{l}12 \\
16\end{array}$ & $\begin{array}{l}0,895 \\
0,918\end{array}$ & $\begin{array}{l}0,271 \\
0,200 \mathrm{NS}\end{array}$ & $\begin{array}{l} \pm 0,078 \\
\pm 0,040\end{array}$ & $0,000.277$ & $\begin{array}{r}6,93 \\
15,07\end{array}$ \\
\hline Moelle épinière & $\begin{array}{l}1-3 \\
4-7\end{array}$ & $\begin{array}{l}12 \\
16\end{array}$ & $\begin{array}{l}0,963 \\
0,914\end{array}$ & $\begin{array}{l}0,509 \\
0,484 \mathrm{NS}\end{array}$ & $\begin{array}{l} \pm 0,081 \\
\pm 0,101\end{array}$ & 0,000645 & $\begin{array}{l}0,180 \\
0,288\end{array}$ \\
\hline Yeux & $\begin{array}{l}1-3 \\
4-7\end{array}$ & $\begin{array}{l}12 \\
16\end{array}$ & $\begin{array}{l}0,878 \\
0,711\end{array}$ & $\begin{array}{l}0,325 \\
0,319 \text { NS }\end{array}$ & $\begin{array}{l} \pm 0,101 \\
\pm 0,047\end{array}$ & 0,001237 & $\begin{array}{l}0,626 \\
0,725\end{array}$ \\
\hline Tube digestif & $\begin{array}{l}1-3 \\
4-7\end{array}$ & $\begin{array}{l}12 \\
16\end{array}$ & $\begin{array}{l}0,945 \\
0,857\end{array}$ & $\begin{array}{l}1,158 \\
0,865 * *\end{array}$ & $\begin{array}{l} \pm 0,022 \\
\pm 0,024\end{array}$ & 0,004196 & $\begin{array}{l}0,012 \\
0,276\end{array}$ \\
\hline Rate & $\begin{array}{l}1-3 \\
4-7\end{array}$ & $\begin{array}{l}12 \\
16\end{array}$ & $\begin{array}{l}0,976 \\
0,574\end{array}$ & $\begin{array}{l}1,722 \\
0,722 * *\end{array}$ & $\begin{array}{l} \pm 0,219 \\
\pm 0,483\end{array}$ & 0,011038 & $\begin{array}{l}0,00005 \\
0,044\end{array}$ \\
\hline Foie & $\begin{array}{l}1-2 \\
3-7\end{array}$ & $\begin{array}{r}8 \\
20\end{array}$ & $\begin{array}{l}0,945 \\
0,957\end{array}$ & $\begin{array}{l}1,122 \\
1,103 \mathrm{NS}\end{array}$ & $\begin{array}{l} \pm 0,313 \\
\pm 0,135\end{array}$ & 0,002526 & $\begin{array}{l}0,0087 \\
0,0082\end{array}$ \\
\hline Reins & $\begin{array}{l}1-2 \\
3-7\end{array}$ & $\begin{array}{r}8 \\
20\end{array}$ & $\begin{array}{l}0,918 \\
0,939\end{array}$ & $\begin{array}{l}1,233 \\
0,790 *\end{array}$ & $\begin{array}{l}+\quad 0,422 \\
+0,118\end{array}$ & 0,002416 & $\begin{array}{l}0,0008 \\
0,034\end{array}$ \\
\hline Surrénales & $\begin{array}{l}1-3 \\
4-7\end{array}$ & $\begin{array}{l}10 \\
16\end{array}$ & $\begin{array}{l}0,528 \\
0,799\end{array}$ & $\begin{array}{l}0,132 \\
0,497 * *\end{array}$ & $\begin{array}{l} \pm 0,043 \\
\pm 0,055\end{array}$ & 0,001745 & $\begin{array}{l}0,042 \\
0,835\end{array}$ \\
\hline Hypophyse & $1-7$ & 28 & 0,742 & 0,657 & $\pm 0,197$ & 0,026340 & 0,060 \\
\hline Thyroïde & $1-7$ & 28 & 0,774 & $0,484 \mathrm{NS}$ & $\pm 0,162$ & 0,011707 & 1,516 \\
\hline
\end{tabular}

$\mathrm{N}=$ Nombre d'animaux.

$r=$ Coefficient de corrélation entre $\log x$ et $\log y$.

Pour $\alpha$ et par rapport au chiffre précédent, différence :

NS Non significative. * Significative $(\mathrm{P}:=0,05)$.

${ }_{*}^{*}$ Très significative $(\mathrm{P}=0,01)$. 
d'assurer aux calculs une meilleure efficacité. Entre les deux (ou trois) hypothèses possibles suggérées dans chaque cas par les graphiques, nous avons retenu celle dont la variance résiduelle est la plus faible. Dans la presque totalité des cas, le calcul ne fait d'ailleurs que confirmer l'impression dominante laissée par le graphique.

Le tableau 6 regroupe les résultats obtenus, retenant dans chaque cas les deux droites de régression auxquelles l'ensemble des données s'ajuste le mieux et que nous considérons comme une estimation des deux droites d'allométrie les plus probables (le tissu gras, déjà étudié plus haut, et dont l'évolution est dissemblable dans les deux sexes n'y figure pas).

Dans ce tableau figurent également l'hypophyse et la thyroïde, bien que leurs données aient été ajustées à une seule droite de régression. Ces organes présentent en effet une très forte variabilité, et la dispersion des points expérimentaux est telle que la variance résiduelle demeure élevée, quel que soit 1'ajustement essayé. Rien ne permet d'ailleurs graphiquement de supposer un changement d'évolution à un stade plutôt qu'à un autre, et l'ajustement à une seule droite, tout en indiquant la tendance générale d'accroissement, est encore ce qui correspond le mieux à l'ensemble des données.

Il n'en est pas de même avec le thymus pour lequel, malgré une assez forte variabilité, la représentation graphique ne laisse guère de doute quant au stade (stade 3 ; $9 \mathrm{~kg}$ de poids vide) à partir duquel débute son involution (fig. II).

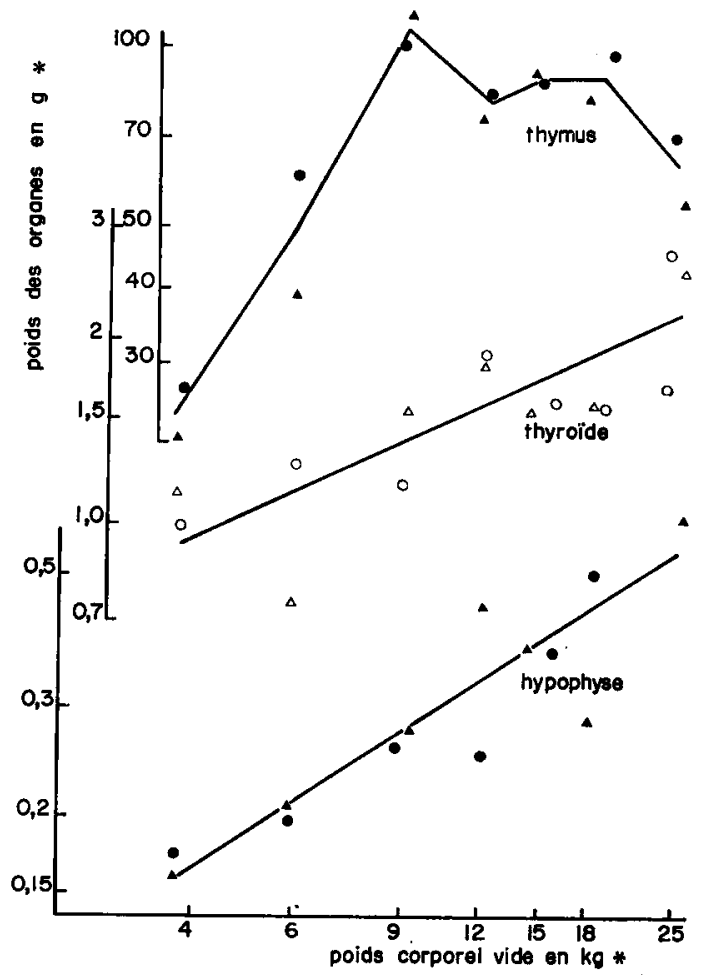

FIG. II. - Évolution de glandes endocrines d forte variabilité $\Delta \Delta$ mâles $\{$ (moyenne de 2 animaux) * Echelle logarithmique 
Les graphiques correspondants aux données du tableau 6 sont représentés aux figures II et I2.

Pour de nombreux tissus ou organes se produit, à partir d'un certain stade, un véritable saut de la droite d'allométrie, correspondant à un rapide et assez brusque changement de la valeur du paramètre $b$ de l'équation fondamentale (I) d'allométrie, et que l'on pourrait appeler, selon l'expression de MEUNIER (I959) cité par GouLD (I966) une " transposition " de la droite d'allométrie.

En fait, pour ces auteurs, le terme " transposition " ne s'applique légitimement que lorsqu'il n'y a pas en même temps changement de pente, les deux droites d'allométrie demeurant parallèles. C'est pourquoi nous luxi préférons le terme de " translation ". Ce n'est que dans ce cas que la modification du paramètre $b$ prend une signification particulière, tout en indiquant le sens de la translation : vers le haut (cerveau, moelle épinière, yeux) ou vers le bas (foie). Lorsque les pentes diffèrent, la valeur de $b$ est évidemment changée, et sa modification peut ne pas correspondre au sens du déplacement de la $2^{\mathbf{e}}$ droite par rapport à la première. Dans ce cas, comme il n'y a pas véritable translation, ni évidemment non plus, "discontinuité " dans la croissance au sens où TEISSIER (I934) a pu employer ce terme avec les Arthropodes, nous utiliserons de préférence le terme de "décalage " entre les droites d'allométrie.

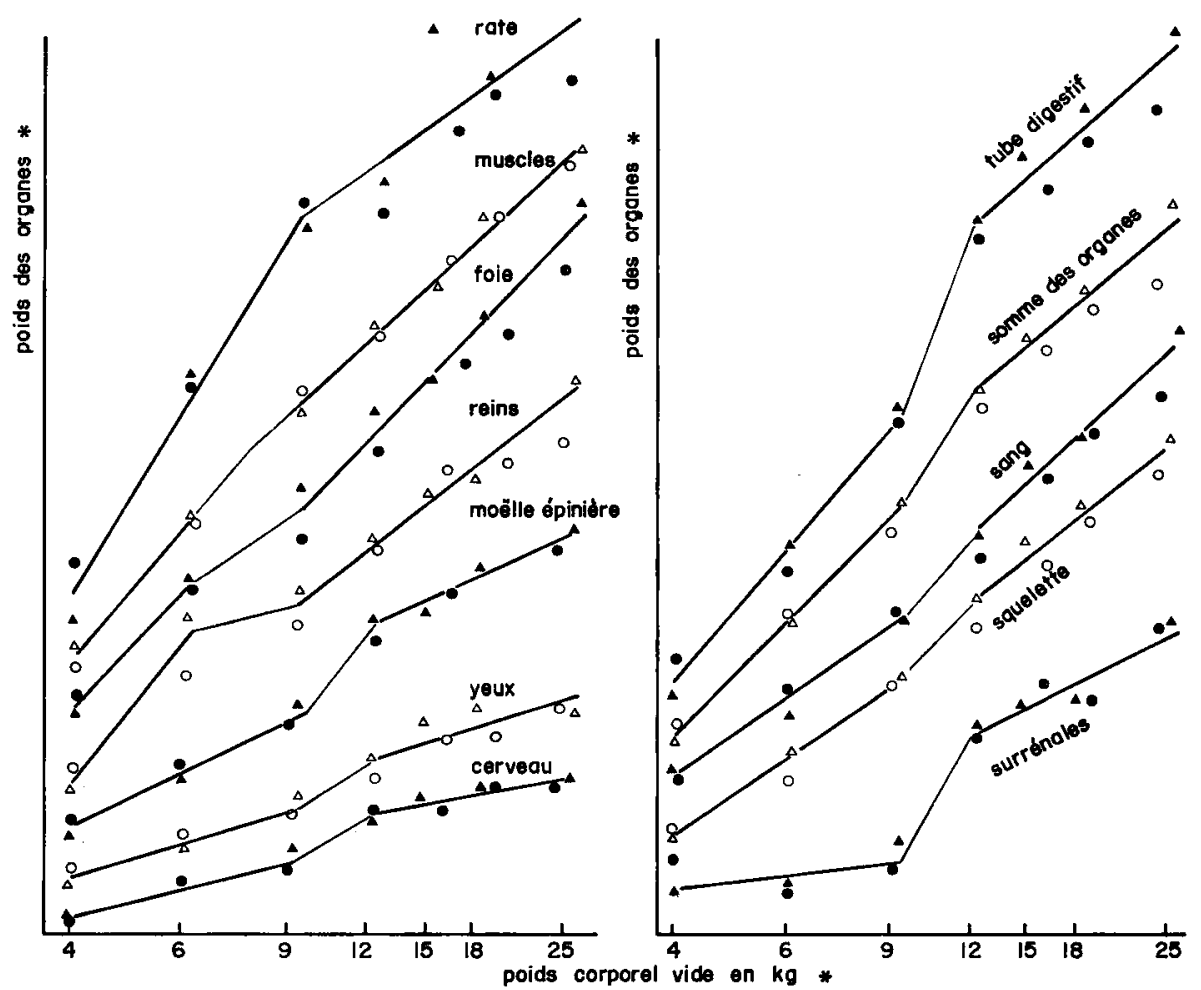

FIG. I2. - Évolution de divers tissus et organes selon deux droites d'allométrie (translations ou décalages)

$\underset{\text { ¿ }}{\Delta \text { femelles }}\{$ (moyenne de 2 animaux) * Échelle logarithmique 
L'ensemble des résultats évoqués dans les paragraphes précédents a été présenté, pour les principales régions étudiées, sous forme d'un schéma synoptique, dans la figure 13 .

Ce schéma, sans représenter cette fois l'évolution pondérale exacte des diffé-

Testicules

Ovoires

Thymus

Hypophyse

Thyroide

Surrénales

Tissus gras $\sigma^{\circ}$

Tissus gras

Reins

Foie

Muscles

Rate

Coour

Tube digestif

$\Sigma$ des organes

Squelette

Sang

Moëlle épinière

Yeux

Cerveau

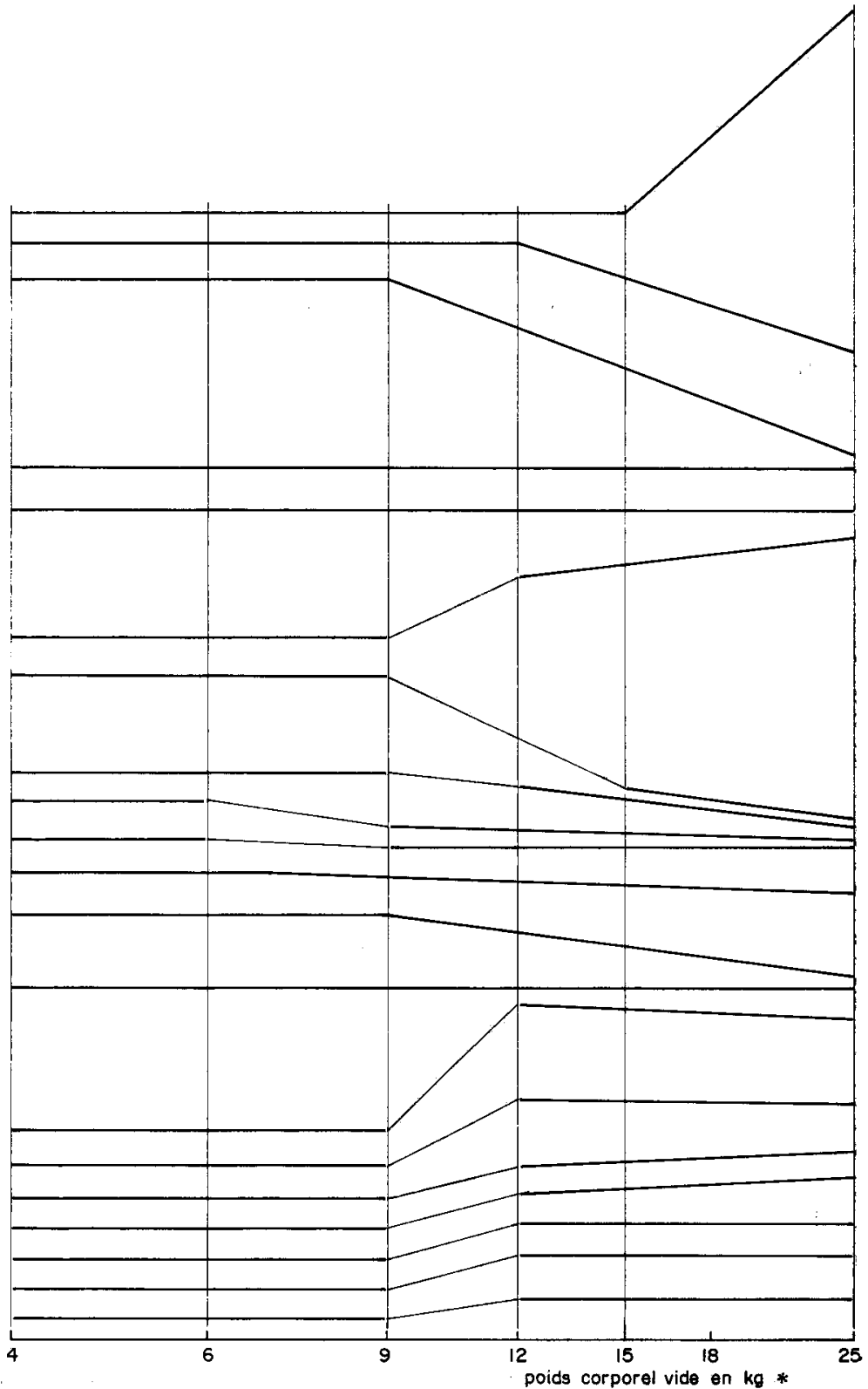

FIG. 13. - Schéma synoptique montrant l'évolution comparée des principaux tissus et organes par rapport à celle des premiers stades post-nataux

* Échelle logarithmique 
rentes régions, cherche, par contre, à traduire optiquement l'allure comparée de leur croissance relative et de ses variations au cours de la période étudiée, par rapport à ce qu'elle est au cours des premiers stades postnataux. Des stades I à 3 (ou éventuellement I à 2) ces régions évoluent selon une certaine droite d'allométrie. Quelle que soit la pente de cette dernière, cette croissance régulière est dans chaque cas représentée par une ligne horizontale. Puis survient un changement d'allométrie, une translation ou un décalage, suivis de l'établissement d'une nouvelle droite d'allométrie. Celle-ci est alors représentée par un trait ascendant si sa pente est supérieure à celle de la première droite, par un trait descendant si elle est inférieure, ou par un trait horizontal si elle a la même pente (translation). L'importance plus out moins grande des décalages ou des changements de pente sont figurés par des traits plus ou moins obliques. Les organes ayant une seule droite d'allométrie tout au long des stades I à 7 sont figurés par une seule ligne horizontale.

\section{DISCUSSION}

\section{I. - Premiers stades postnataux (1-3)}

Les différences de valeur discutées dans ce paragraphe entre certains coefficients d'allométrie ont été testées quant à leur signification statistique (tab1. 4). L'ensemble des résultats demeure conforme aux conclusions déjà déposées par d'autres auteurs (HAmmond, I932; PALsson et Verges, I952 $a$; PALSSON, I955; WALIACE, I948) à savoir que les tissus ou organes ayant bénéficié d'un développement prioritaire durant la vie prénatale sont après la naissance ceux dont la vitesse de croissance relative est la plus lente. Se trouve ainsi confirmé, en précocité décroissante, l'ordre de succession suivant : tissu nerveux, squelette, muscles, tissus gras. Cet ordre se retrouve d'ailleurs chez la plupart des autres espèces domestiques étudiées : bovins, porcs, volailles (pour 1a littérature, voir notamment PALSson, I955). Plus récemment chez le Lapin, CANTIER et al., (I969) trouvent également un ordre de succession analogue, avec certains coefficients d'allométrie identiques aux nôtres (cerveau : 0,27 , muscles : I,I5) et d'autres un peu différents.

Pour ces divers auteurs, le tissu nerveux est généralement représenté par le seul cerveau. Cependant nos résutats montrent que la moelle épinière présente un taux d'accroissement relatif très significativement plus élevé, tout en demeurant significativement inférieur à celui du squelette. Ce fait est probablement en liaison avec le développement relatif en longueur du corps et l'allongement de la colonne vertébrale.

$D^{\prime}$ autre part les yeux (tabl. 6) semblent évoluer à une allure intermédiaire entre celles du cerveau et de la moelle épinière bien que leur coefficient d'allométrie ne diffère pas significativement de celui du cerveau. Ce résultat correspond également à celui de PALSSON et VERGES (I952 a) qui avaient déjà signalé une croissance relative des yeux un peu plus rapide que celle du cerveau.

$\mathrm{Ne}$ sont pas significatives les différences entre les coefficients d'allométrie du squelette et du sang d'une part, de la somme des organes (non compris le système nerveux) et la somme des muscles d'autre part.

En résumé, les éléments de la succession suivante : cerveau, moelle épinière, 
squelette et sang, muscles et organes, tissus gras, présentent des coefficients d'allométrie qui diffèrent entre eux significativement ou très significativement.

Pour les graisses, PALSSON et VERGES (I952 a) avaient déjà observé un accroissement relatif moins rapide du gras intermusculaire que du gras de couverture. Nous y ajoutons auparavant le gras interne dont la vitesse de croissance relative est encore moindre. Les coefficients d'allométrie du gras interne, du gras intermusculaire et du gras de couverture croissent respectivement dans cet ordre et diffèrent entre eux très significativement. Il en est de même pour ceux des dépôts adipeux péritonéaux du " ratis " et de la " toilette ".

Bien que l'ordre de succession constaté dans la rapidité de croissance relative pour les principaux tissus ou organes apparaisse comme très général quand on les considère dans leur ensemble, il peut cependant présenter des exceptions si l'on considère certains organes ou régions anatomiques individuels. Ainsi le muscle cardiaque (dont le taux d'accroissement relatif est d'ailleurs constant) a un coefficient d'allométrie qui diffère significativement de celui de l'ensemble des muscles du squelette. Mais il en est de même de celui du muscle squelettique extenseur antérieur du métacarpe. De même aussi celui du sternum diffère très significativement de celui du squelette entier (tabl. 4).

Ainsi contrairement à ce qui se passe pour les tissus correspondants pris dans leur tout, le sternum qui est un os, a un coefficient d'allométrie plus élevé que celui de certains muscles, tel l'extenseur antérieur du métacarpe. Cela peut d'ailleurs s'expliquer si l'on s'en rapporte aux notions de "gradients de croissance " évoqués par les auteurs déjà cités. Le muscle extenseur antérieur du métacarpe fait partie d'une région à développement précoce, dont la croissance postnatale doit donc être moins rapide que celle des régions à développement plus tardif, comme la cage thoracique, à laquelle appartient le sternum. Il faut donc éventuellement tenir compte de ces notions et ne pas identifier sans examen la croissance d'une partie individuelle d'un tissu avec celle du tissu dans son ensemble.

Il est assez malaisé de comparer les valeurs que nous indiquons pour les coefficients d'allométrie à celles que 1'on peut relever dans la littérature, ces dernières étant assez rares et généralement obtenues dans des conditions dissemblables.

ELSLEY et al. (I964) reprenant les données de PAISSON et VERGES (I952) calculent pour les principaux tissus de la carcasse les coefficients d'allométrie non par rapport au poids vide mais par rapport à la somme (os + muscles) qu'ils prennent comme approximation du poids de carcasse "dégraissé ". Nous avons ramené leurs résultats en estimation par rapport au poids vide en les multipliant par le coefficient 0,87 qui représente la pente de la droite de régression de la variable $\mathrm{L}, \mathrm{og}$ (os + muscles) sur la variable Log (poids vide), calculée d'après leurs données. Dans ces conditions leurs coefficients d'allométrie deviennent : squelette 0,65 ; muscles 0,96 ; gras intermusculailaire, I,58; gras de couverture I,87. Les deux premiers sont du même ordre que les nôtres, bien qu'un peu plus faibles; les suivants sont beaucoup plus faibles, bien que respectant 1'ordre de succession.

Cette différence s'explique du fait que nos données correspondent aux premiers stades postnataux (o à 25 jours) où d'une façon générale la plupart des tissus ont un développement relatif plus rapide que dans les périodes qui suivent. Le plus grand nombre de données de ELSLEY et al. se rapportent à des animaux allant de 2 mois à Io mois. 
BOCCARD et al. (I964) rapportent des résultats pour différentes vitesses de croissance globale dont l'une (225 g par jour, entre o et 75 jours) est comparable à celle de nos agneaux jusqu'au $3^{\mathbf{e}}$ stade. Les coefficients d'allométrie qu'ils indiquent dans ces conditions sont respectivement 0,76 pour le squelette et $I, I 6$ pour les muscles, valeurs très proches des nôtres et comprises dans les limites de nos intervalles de confiance. Pour l'ensemble du tissu gras ils indiquent la valeur $\mathrm{I}, 8$ qui est nettement moindre que la nôtre $(2,8)$. Il faut toutefois observer que la période étudiée va de o à 75 jours, ce qui correspondrait pour nos agneaux aux stades I à 5. Or, entre les stades 3 et 5 la vitesse d'évolution du tissu gras diminue fortement surtout chez les mâles. Si l'on calculait une droite de régression pour l'ensemble de la période I à 5 , on trouverait une valeur voisine de $I, 8 \mathrm{du}$ même ordre que celle indiquée par BocCARD et al. Les agneaux étudiés par ces auteurs sont de la race Préalpes $d u$ Sud.

Cette comparaison avec les nôtres, de race Mérinos d'Arles, est intéressante en ce qu'elle montre que les principaux tissus de la carcasse ont, chez deux races différentes, mais de formats adultes assez voisins, des taux comparables de croissance relative, lorsque les agneaux ont une même vitesse de croissance globale. Bien qu'on puisse penser qu'il en serait probablement de même avec certaines autres races de format adulte comparable, cette seule observation ne permet toutefois aucune généralisation.

Nos résultats sur cette courte période postnatale sont donc dans l'ensemble conformes à ceux obtenus par d'autres auteurs sur des périodes généralement plus étalées, tout en apportant un certain nombre d'informations et de précisions complémentaires.

\section{2. - Inégalités de développement selon le sexe}

Durant la période étudiée (o-I6o jours) nous avons comparé des animaux, qui tant mâles que femelles, ont suivi une même courbe de croissance globale.

Certains tissus ou organes ont évolué de façon analogue chez les deux sexes. Parmi ceux-ci on trouve notamment ceux ayant une fonction vitale fondamentale et dont le développement est précoce (tissu nerveux) ou assez précoce (ccur, sang). On conçoit que le sexe, dont les manifestations majeures sont plus tardives, les influence peu et que leur importance relative soit donc comparable dans les deux sexes.

Certaines des différences relevées proviennent vraisemblablement de différences de conformation liées au sexe (muscles du cou, os de la tête, ensemble du squelette).

D'autres sont à rapporter à des différences de métabolisme s'établissant plus ou moins précocement. En particulier le foie rapidement plus lourd chez les mâles, et l'avance progressive prise par les organes, notamment les organes digestifs, par rapport à ceux des femelles, semblent correspondre aux besoins d'un métabolisme progressivement plus intense chez les mâles. Cette avance se précise d'ailleurs à partir d'un stade (stade 3 ; $9 \mathrm{~kg}$ de poids vide) où ces derniers subissent une mobilisation importante de leurs réserves graisseuses, alors que les femelles en poursuivent toujours l'accumulation.

A partir de ce stade, les femelles compensent le moindre poids de leurs organes et du squelette par un poids supérieur en tissu gras. D'autre part, le développement musculaire étant égal chez les deux sexes, les femelles devraient avoir après le stade 3 un poids de carcasse légèrement supérieur à celui des mâles, pour un même poids vide. C'est ce que l'on peut constater graphiquement (fig. I4). 
Ainsi pour une même croissance entre 25 et I6o jours, s'étalant entre ro $\mathrm{kg}$ et $30 \mathrm{~kg}$ de poids vif, les agneaux femelles ont un rendement en carcasse un peu plus élevé, un squelette un peu plus fin, autant de muscles mais davantage de gras que les agneaux mâles. Du point de vue de la boucherie, les deux premiers de ces éléments sont favorables, le dernier pouvant l'être moins selon le pourcentage de gras souhaité. Ces résultats sont conformes à ceux également relevés par PALSSON et VERGES ( $195^{2} b$ ) sur des agneaux élevés en haut niveau de nutrition, à l'âge de 9 semaines et au poids vide de $24 \mathrm{~kg}$ (moindre rendement en carcasse pour les mâles, davantage d'os et moins de graisse). Ils étaient d'ailleurs par la suite inversés chez des animaux de 4 I semaines, mais les auteurs n'étudiaient aucun stade intermédiaire.

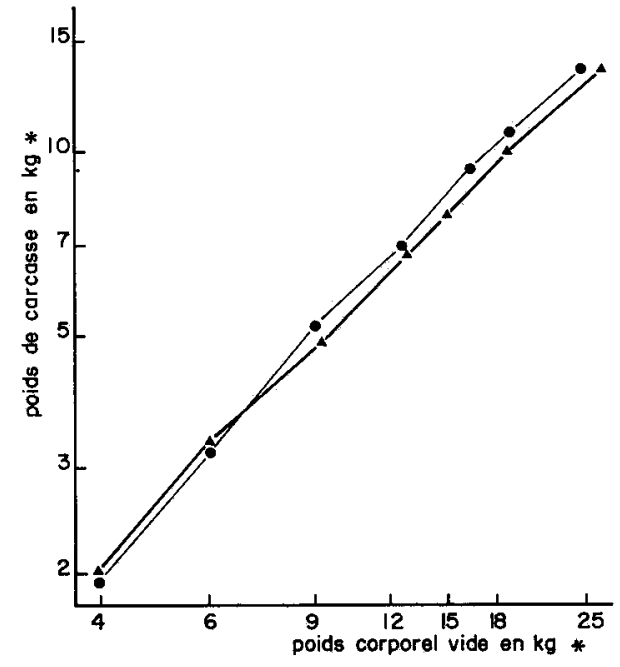

FIG. I4. - Évolution du poids de carcasse en fonction du poids vide

- mâles i (moyenne de 2 animaux)

- femelles

* Échelle logarithmique

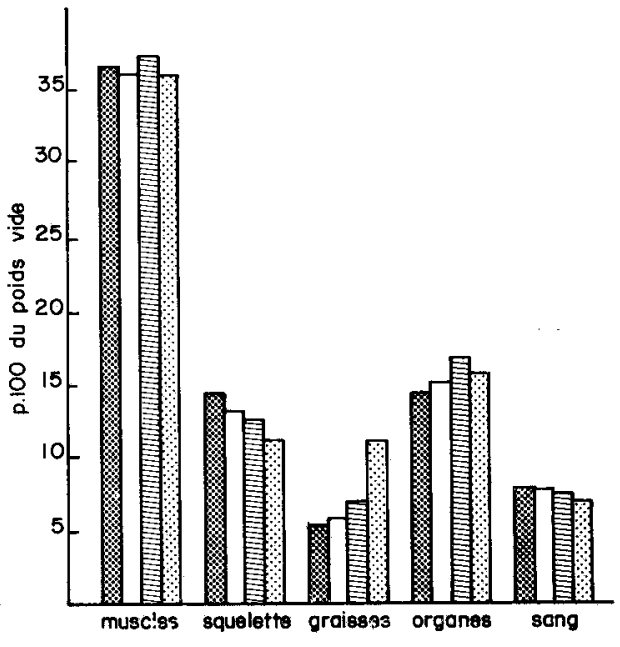

FIG. I5. - Composition corporelle chez les deux sexes, aux stades 2 (poids vide $6 \mathrm{~kg}$ ) et 4 (poids vide $\mathrm{I} 2 \mathrm{~kg}$ ) stade 2
$\square$
0

L'ensemble de ces comparaisons et l'examen des graphiques correspondants montrent que jusqu'à un poids vif d'environ $\mathrm{to} \mathrm{kg}$, le sexe n'influence que très peu la croissance relative des agneaux. Son influence n'est cependant pas nulle, et se manifeste d'ailleurs dès l'époque prénatale, puisquele poids moyen de naissance des agneaux mâles du troupeau est significativement supérieur à celui des femelles (PRUD'HON et al., Ig68).

Jusqu'à Io $\mathrm{kg}$ la composition corporelle est très proche et évolue parallèlement chez les deux sexes, bien que certaines tendances différentielles se manifestent, discrètement mais visiblement (dépôts adipeux tous très peu mais systématiquement plus élevés chez les femelles, et squelette un peu plus léger). Au-delà, des différences s'amorcent ou s'accusent, traduisant une influence grandissante du sexe, bien que les gonades ne modifient qu'un peu plus tard leur évolution pondérale (stades 4 ou 5). 
La figure $I_{5}$ et le tableau 7 illustrent ces principales différences de la composition corporelle en exprimant le pourcentage du poids vide que représentent les principaux tissus et organes à deux stades encadrant le stade 3 .

TABLEAU 7

Composition corporelle aux stades 2 et 4

\begin{tabular}{|c|c|c|c|c|c|}
\hline & & \multicolumn{2}{|c|}{ Stade 2} & \multicolumn{2}{|c|}{ Stade 4} \\
\hline & & $\delta$ & ๆ & 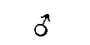 & $q$ \\
\hline \multicolumn{2}{|l|}{ Age (jours) } & 12 & 11 & 50 & 51 \\
\hline \multicolumn{2}{|c|}{ Poids "vide " $(g) \ldots \ldots \ldots$} & 6000 & 6003 & 12360 & 12440 \\
\hline \multirow{6}{*}{$\begin{array}{c}\text { Pourcentage } \\
\text { du } \\
\text { poids } \\
\text { vide }\end{array}$} & Muscles .... & 36,3 & 35,8 & 36,9 & 35,7 \\
\hline & Squelette ... & 14,1 & 12,9 & 12,2 & 10,9 \\
\hline & Graisses $\ldots$ & 4,9 & 5,3 & 6,5 & 10,7 \\
\hline & Organes ... & 14,3 & 14,6 & 16,4 & 15,5 \\
\hline & Sang ....... & 7,2 & 7,2 & 7,0 & 6,7 \\
\hline & Total ....... & 76,8 & 75,8 & 79,0 & 79,5 \\
\hline
\end{tabular}

Chaque colonne correspond à la moyenne de 2 animaux.

On voit que les pourcentages des tissus musculaire et sanguin n'ont pratiquement pas varié et sont d'autre part demeurés très proches dans les deux sexes.

Le pourcentage du squelette a diminué, mais dans les mêmes proportions, et il demeure plus élevé chez les mâles. Par contre, celui des organes et surtout celui des matières grasses ont augmenté. Mais alors que les organes ont progressé sensiblement plus chez les mâles, c'est chez les femelles que le tissu gras a beaucoup plus progressé.

Le graphique permet d'autre part d'observer nettement une évolution exactement inverse du squelette et du tissu gras. Cette sorte d'antagonisme de développement entre deux tissus chez lesquels rien n'indique apparemment un rapport direct peut être intéressante à relever, sans pouvoir toutefois en tirer pour l'instant aucune conclusion.

Une objection pourrait être faite ici. La croissance pondérale des femelles, à une allure un peu supérieure à celle de la croissance moyenne de leur sexe, pourrait être cause d'une certaine accumulation de gras supplémentaire, et inversement pour les mâles. Il est certain qu'une telle objection serait valable à des stades beaucoup plus avancés, où les différences pondérales moyennes entre les deux sexes deviennent très importantes. Mais entre les stades 3 et 4 , où l'évolution des matières grasses s'inverse brutalement chez les mâles, ceux-ci, comme les femelles et même davantage, sont encore très proches de la courbe de croissance moyenne de leur sexe (fig. I). La croissance pondérale globale, très légèrement ralentie par rapport aux stades précédents, ne peut donc être ici la cause majeure des importantes différences constatées.

Il est certain que chaque sexe évolue sous la dépendance d'un équilibre endocrinien quilui est propre. Or, à ces stades encore précoces du développement, et dans des 
conditions analogues de nutrition, de croissance globale et d'environnement, les facteurs intrinsèques (et en particulier les facteurs endocriniens) sont évidemment les principaux responsables des différences d'évolution de la croissance relative. Les résultats de notre analyse suggèrent l'hypothèse d'un équilibre hormonal très voisin chez les deux sexes - tout au moins dans ses effets - jusqu'aux environs de ro $\mathrm{kg}$ de poids vif. A partir de ce stade s'établirait assez rapidement un nouvel équilibre, plus nettement différent pour chaque sexe, entraînant des modifications plus ou moins importantes et parfois différentes de la croissance relative. Les nombreux changements d'allométrie constatés à partir de ce stade ne peuvent d'ailleurs que renforcer ce point de vue.

\section{3. - Évolution de la croissance relative des différentes parties}

a) Principaux tissus et organes.

Parmi les organes, en nombre assez limité, qui montrent un taux d'accroissement relatif constant, correspondant à une seule droite d'allométrie, certains (hypophyse, thyroïde) ont une très forte, d'autres (cœur, os canons) une faible variabilité. Les chiffres de variance résiduelle pour la thyroïde ou l'hypophyse sont de l'ordre de Io à I 5 fois plus élevés que ceux concernant le cœur ou les os canons (tab1. 5 et 6).

Dans le cas de ces derniers organes, compte tenu d'un intervalle de confiance assez étroit, une détermination assez précise du coefficient d'allométrie peut être obtenue, qui serait encore améliorée par un plus grand nombre d'animaux (entre 0,74 et 0,84 pour le cour ; entre 0,50 et 0,55 pour les os canons, tabl. 5).

De tels organes à évolution continue et à faible variabilité pourraient valablement être utilisés comme organes de référence pour des études comparatives. Le cour et l'os canon ont d'ailleurs été déjà proposés par divers auteurs à cette fin. On pourrait y ajouter les phalanges et même, pour des études comparées d'évolution musculaire, l'ensemble des muscles $d u$ cou (tabl. 5). Notons encore l'éventuel intérêt du sternum jusqu'au stade 3, seul organe à évoluer jusque-là selon une exacte isométrie (tab1. 4).

Pour la thyroïde et 1'hypophyse, la valeur calculée du coefficient d'allométrie n'est que largement indicative, entachée par un intervalle de confiance élevé (tabl. 6). D'ailleurs les valeurs correspondantes de $\alpha$ ne diffèrent pas significativement. Toutefois le taux d'accroissement relatif de l'hypophyse demeure très significativement plus élevé que celui du cerveau. CANTIER et al. (I969) étudiant chez le Lapin les divers ajustements possibles, trouvent également pour la thyroïde et 1 'hypophyse une seule droite d'allométrie (alors qu'i1 en existe, comme chez 1'Agneau, deux pour les surrénales) et la pente de celle de l'hypophyse est également significativement plus élevée que celle du cerveau.

D'autre part, un petit nombre d'organes (peau, maxillaire inférieur) semblent ne pas obéir à une relation simple d'allométrie, et correspondent en coordonnées logarithmiques à un tracé curviligne. Une telle possibilité, bien que paraissant peu fréquente, a été signalée notamment par GouLD (I966) qui cite divers exemples de tendances curvilignes empruntés à différents auteurs, et par LAIRD (I965) qui se rapporte à une démonstration de LUMER (I937).

Cependant la plupart des tissus ou organes étudiés présentent des relations d'allométrie, avec à certains stades changement de pente, translation ou décalage de la droite d'allométrie. 
La représentation schématique de la figure I3 rassemblant synoptiquement les principaux résultats, met immédiatement en évidence la grande fréquence des changements survenant au niveau du stade 3 (25 jours; $9 \mathrm{~kg}$ de poids vide), bien que certains soient plus précoces (reins, foie, muscles) d'autres plus tardifs (gonades). Beaucoup de ces changements consistent en une brusque accélération de l'évolution pondérale relative entre les stades 3 et 4 , suivie de l'établissement d'une nouvelle droite d'allométrie entre les stades 4 à 7 . Les deux droites d'allométrie I-3 et 4-7 ont parfois subi ce que nous avons appelé un décalage, avec des pentes significativement différentes, soit en augmentation (surrénales) soit en diminution (tube digestif). Mais plus souvent elles sont pratiquement parallèles ou ne diffèrent pas significativement quant à leur pente (tabl. 6), correspondant ainsi à une translation qu'on peut dire positive ou vers le haut (cerveau, yeux, moelle épinière, sang, squelette, somme des organes) (fig. I3).

D'autres organes ou tissus subissent une évolution inverse : décalage vers le bas (tissu gras chez les mâles ; reins) ou translation négative (foie). La translation pour le foie ou le décalage pour les reins s'établissent plus précocement, entre les stades 2 et 3 . Signalons encore que chez le lapin CANTIER et al.(I969) trouvent pour le foie (dans les deux sexes) et pour les reins (mâles) une évolution analogue : deux droites d'allométrie avec un décalage vers le bas.

Il peut aussi y avoir diminution du coefficient d'allométrie, sans décalage apparent (tissu gras chez les femelles, fig. 8 a). Dans le cas du thymus, il y a régression à partir du stade 3 (fig. II). Pour la rate, les deux droites d'allométrie calculées, dont les pentes diffèrent très significativement, se recoupent également aux environs du stade 3 (fig. I2) alors que dans le cas des muscles elles se recoupent au voisinage de $7 \mathrm{~kg}$ de poids vide ou un peu plus (fig. I2) correspondant à $7,500 \mathrm{~kg}$ à $8 \mathrm{~kg}$ de poids vif, c'est-à-dire à un âge compris approximativement entre $\mathrm{I} 6$ et 20 jours.

On peut se demander si, de même que l'accélération rapide du poids testiculaire correspond au démarrage d'une phase d'intense multiplication cellulaire (COUROT, r962), la baisse du coefficient d'allométrie du muscle ne pourrait correspondre à une phase d'arrêt ou au moins de fort ralentissement de cette multiplication.

On admet encore souvent que la croissance postnatale du muscle résulte presque uniquement de 1 'hypertrophie des fibres préexistantes. Il semble toutefois que même si les fibres ne croissent plus en nombre, elles subissent encore pendant une période assez prolongée des multiplications nucléaires qui correspondent à une véritable hyperplasie cellulaire du muscle, qu'on peut distinguer de la multiplication des fibres. proprement dite. Cependant pour divers auteurs cités par JouBERT (I956) il y a encore multiplication des fibres au moins au début de la vie postnatale, jusqu'à un stade qui reste encore à préciser. Il existe d'ailleurs certainement des différences selon les espèces, et peut-être selon les muscles étudiés, ainsi qu'éventuellement selon le sexe ou le niveau de nutrition. Pour Goldspink (I962) étudiant la souris albinos, le nombre de fibres du biceps brachial augmente après la naissance jusqu'à ce que l'animal ait atteint un poids de $8 \mathrm{~g}$. HrkAmI et MIzUNo (1965) trouvent que chez les poussins de deux races différentes le nombre de fibres augmente pendant 4 à 6 semaines après l'éclosion pour le muscle pectoral, alors qu'il semble ne pas varier pour le sartorius.

DURAND et al. (1965), grâce à des tests biochimiques fondés sur la mesure de l'ADN, étudient d'autre part l'influence respective sur la croissance de l'hyperplasie 
_et de l'hypertrophie cellulaires. Ils montrent que pour la carcasse éviscérée du rat mâle (Wistar), dont les muscles représentent la majeure partie, 1'hyperplasie est le facteur principal de la croissance jusqu'à 7 semaines, le grandissement l'emportant par la suite. Ces mêmes auteurs ont également montré (I967) qu'une restriction alimentaire énergétique tend à conserver plus loin dans le temps le pouvoir hyperplasique du muscle. Utilisant des techniques analogues pour les muscles des membres du Lapin, CORDESSE (I968) montre que chez les deux sexes la multiplication nucléaire l'emporte sur le grandissement jusqu'à 60 jours, les deux processus ayant ensuite jusque vers $\mathrm{r} 20$ jours un rôle équivalent chez les mâles, alors que le premier demeure encore prépondérant chez les femelles. Or, CANTIER et al. (rg69) situent chez le Lapin le changement d'allométrie musculaire à partir de $2200 \mathrm{~g}$ de poids vide, soit à environ I 2 semaines, c'est-à-dire entre 60 et I20 jours. I1 se pourrait de même que chez l'Agneau, animal dont la naissance se situe à un stade physiologiquement plus avancé, 1e changement d'allométrie des muscles constaté aux environs de 20 jours corresponde sinon à l'arrêt véritable de la multiplication des fibres, du moins à un ralentissement marqué de l'hyperplasie musculaire. C'est évidemment là une simple hypothèse qui demanderait vérification.

Ainsi, entre les stades 3 et 4 ou un peu plus précocement, deux séries de tissus ou organes accusent assez nettement une évolution contraire. Les uns subissent une accélération passagère plus ou moins intense de leur taux d'accroissement, suivie de l'établissement d'une nouvelle droite d'allométrie dont la pente est selon les cas supérieure, égale ou inférieure à celle de la précédente (tube digestif, surrénales, squelette, sang, yeux). Le système nerveux lui-même, souvent considéré comme particulièrement stable dans son évolution, enregistre cette accélération (cerveau, et plus nettement encore moelle épinière). D'autres, en compensation, ralentissent leur évolution (muscles, rate, dans les deux sexes ; matières grasses chez les femelles) ou voient leur droite d'allométrie transposée vers le bas (reins, foie) ou enregistrent même une véritable régression (matières grasses chez les mâles, thymus).

Cet ensemble de changements encadrant le stade 3 fait évidemment apparaître celui-ci comme un point critique de la croissance, où surviennent d'importantes modifications du métabolisme vraisemblablement liées à des variations d'équilibres hormonaux. Ces observations sont également en accord avec celles de Boccard, Dumont et LEFEBVRE ( 1962 ). Ces auteurs, étudiant la croissance relative des régions corporelles chez l'Agneau, avaient trouvé pour certaines régions comme le membre postérieur, un changement d'allométrie à partir d'un poids de $3,500 \mathrm{~kg}$ de la demi-carcasse. Ceci correspond approximativement à $\mathrm{I} 4 \mathrm{~kg}$ de poids vif, soit environ $\mathrm{I} 2 \mathrm{~kg}$ de poids vide, c'est-à-dire un poids correspondant à notre stade 4 (les animaux étudiés étaient des agneaux mâles de la race Ile-de-France, d'un format un peu supérieur à celui du Mérinos d'Arles).

Tout semble donc indiquer qu'à partir du stade 4 un nouvel équilibre hormonal stable est établi, entraînant pour la croissance relative de nouvelles droites d'allométrie. Il semble alors intéressant d'examiner en particulier l'évolution des glandes endocrines.

\section{b) Évolution des glandes endocrines.}

Le cas des gonades a déjà été évoqué. Rien ne permet d'y déceler dès le stade 3 un changement d'évolution, celui-ci étant plus tardif. Le testicule en particulier ne 
subit qu'au-delà du stade $5(\mathbf{I} 5,500 \mathrm{~kg}$ de poids vide; environ $\mathrm{I} 9 \mathrm{~kg}$ de poids vif ; 82 jours) sa brusque accélération d'accroissement.

Cette observation est sensiblement en accord avec celle établie par Courot (I962) sur agneaux Ile-de-France : brusque accroissement du testicule, à partir d'un poids vif d'environ $20 \mathrm{~kg}$, vers 88 jours. CoUroT, dont les conclusions sont confirmées par celles de SkinNer et al. (I968) relatives à l'agneau Suffolk, a montré que cette accélération de croissance du testicule correspond au démarrage de la spermatogénèse, caractérisée en particulier par une multiplication cellulaire intense, et qu'elle est davantage en liaison avec le poids corporel qu'avec l'âge des animaux. Si le développement pondéral du testicule ne semble pas influencé par les facteurs qui entraînent les différentes autres modifications constatées au stade 3, on ne peut cependant affirmer à l'inverse que les hormones androgènes n'ont aucune part à ces modifications car elles sont déjà présentes à la naissance, ainsi que le confirment Skinner et al. (rg68).

En ce qui concerne 1'ovaire, son évolution pondérale semble de même se faire jusqu'au stade 4 à un rythme soutenu, sans rapport apparent avec les autres modifications corporelles plus précoces. Il est toutefois curieux et semble assez paradoxal d'enregistrer par la suite une certaine régression de l'ovaire, jusqu'au stade 7 inclus, ou du moins un arrêt de croissance temporaire de l'organe, compte tenu de la marge possible de variabilité. L'explication ne nous en apparait pas clairement. On peut toutefois observer que les femelles disséquées à partir du stade 5 l'ont été entre fin janvier et fin avril, c'est-à-dire en période de jours croissants, généralement défavorable chez les ovins à l'activité gonadique.

Les divers changements de la croissance relative qui s'établissent en particulier entre les stades 3 et 4 , et qui traduisent des modifications du métabolisme, pourraient semble-t-il être accompagnés à la même période d'un changement d'évolution pondérale de certaines glandes endocrines, et notamment de 1'hypophyse et de la thyroïde. Or ces deux dernières, malgré leur forte variabilité, évoluent selon une allométrie apparamment constante (fig. II). Mais il peut ne pas en être de mêtme de leur niveau de sécrétion ou de la sensibilité des différents tissus à leur action.

Ainsi avons-nous observé que seul parmi les différents dépôts adipeux le gras mésentérique continue à s'accroître rapidement après le stade 3 sans montrer chez les mâles le moindre signe de fléchissement. Cette observation est à rapprocher de calles de KNOBIL et de RESHEF et SHAPIRo cités par WEIL (I965) : à partir de gras épididymaire incubé de rats hypophysectomisés traités à la STH, KNOBIL constate une augmentation des acides gras libres dans le milieu, alors que RESHEF et SHAPIRO opérant de même mais à partir de gras mésentérique n'obtiennent aucune mobilisation. Ce dernier tissu ne semble donc pas sensible à l'action de la STH et il pourrait en être de même chez l'Agneau où la sensibilité à la STH des différents dépôts graisseux pourrait être plus ou moins grande, peut-être selon la nature de ces dépôts ou celle de 1'équipement enzymatique correspondant.

D'autre part, une mobilisation des graisses plus intense et une synthèse protéique supérieure chez les mâles (ensemble des organes en développement plus rapide, foie plus lourd) pourrait correspondre chez eux à un taux plus élevé de STH et de TSH. De même une sécrétion accrue d'ACTH pourrait venir renforcer la mobilisation du tissu gras, tout en provoquant une accélération de la croissance pondérale des surrénales nettement enregistrée entre les stades 3 et 4 (tabl. 6 ; fig. I2). 
Il ne paraît pas invraisemblable qu'il y ait ainsi dans le temps des mises en jeu successives des diverses stimulines hypophysaires, suivies ensuite de modulations variées de leurs actions réciproques. La STH, présente très tôt chez le fœetus, pourrait chez l'agneau nouveau-né n'avoir pas encore une forte activité de croissance, comme cela semble être le cas chez d'autres espèces. Ainsi le Rat hypophysectomisé dans les premiers jours qui suivent sa naissance montre une croissance pondérale presque normale jusqu'à 28 jours, puis très ralentie ensuite (WALKER et al., I952). De même l'hypophysectomie chez le Lapin n'exerce une action nette et immédiate d'arrêt de la croissance que si elle est pratiquée à partir de l'âge de Ioo jours (VEZINHET, I968 b). Par contre, ce dernier auteur, pratiquant chez l'Agneau des hypophysectomies à 25, 5 e et roo jours, obtient dans tous les cas un ralentissement de croissance extrêmement marqué (VEZINHET, I968 $a$ ).

D'autre part BIRGE et al. (I967) ont établi par dosages radioimmunologiques que le contenu comme la concentration hypophysaires en STH diffèrent chez le Rat selon le sexe (teneurs progressivement plus élevées chez les mâles) mais seulement à partir de la $8^{\mathbf{e}}$ semaine, et que ces taux sont modifiables sous l'influence d'hormones sexuelles.

La confrontation de ces diverses observations et de nos résultats peut inciter à penser que l'Agneau pourrait lui-même n'être que peu sensible à l'action de la STH jusqu'aux environs de 25 jours, l'équilibre endocrinien pouvant être jusque-là assez voisin chez les mâles et les femelles, induisant une croissance relative très proche dans les deux sexes, comme celle qui a été constatée. Le taux de STH pourrait ensuite s'accroître davantage chez les mâles, en même temps que pourraient alors entrer successivement en jeu d'autres stimulines hypophysaires comme TSH et ACTH, à des taux éventuellement différents chez les deux sexes et potentialisant peut être l'action de la STH, puis les stimulines gonadiques.

Un tel schéma, suggéré par certains aspects de la croissance relative, demeure encore très hypothétique et très sommaire. Il reste cependant également compatible avec des résultats obtenus par VEZINHET et DAUzIER (I970) sur agneaux mâles hypophysectomisés à 25 jours, certains ayant reçu jusqu'à Ioo jours des injections répétées de STH bovine. Alors que les animaux hypophysectomisés voient leur croissance pondérale très ralentie et s'accroître uniquement par accumulation de tissu gras, les animaux hypophysectomisés mais injectés de STH ont une croissance globale, musculaire et osseuse proche de celle des témoins ; par contre, ils ont subi une sévère régression de leurs réserves graisseuses (analogie avec nos agneaux mâles entre les stades 3 et 5). D'autre part leurs surrénales et testicules régressent presque autant que chez les hypophysectomisés non injectés, alors que ces glandes se développent normalement chez les témoins. Ce sont donc bien d'autres stimulines que la STH qui ont été responsables de l'accroissement surrénalien et testiculaire des animaux témoins, alors que la S'TH était responsable de l'accroissement osseux et musculaire, en même temps que de la mobilisation des graisses, chez les animaux hypophysectomisés injectés.

Un autre effet de la STH enregistré sur agneaux par VEZINHET (I969) concerne le thymus. Alors que l'hypophysectomie entraîne une très forte régression de ce dernier, 1'injection de STH à des animaux hypophysectomisés permet au thymus de se maintenir ou de ne décroître que lentement, comme chez les animaux normaux. Cette légère régression s'accorde d'ailleurs bien avec celle que nous avons enregistrée graphiquement (fig. II). Le thymus, en accroissement d'abord rapide jusqu'au stade 3 (25 jours) atteint à ce moment son développement maximum, à partir duquel il 
amorce sa régression. On peut penser après les observations de VEzINHET, que l'allure lente de celle-ci est conservée un certain temps grâce à l'action de la S'TH.

Toutefois, si le thymus semble particulièrement sensible à l'action de l'hormone de croissance, son accroissement rapide dans les premiers stades du développement s'accorderait assez mal avec notre suggestion précédente d'une moindre sensibilité des tissus à la STH jusque vers 25 jours, à moins que jusqu'à ce stade la STH ne soit pas seule en cause et que le thymus, comme d'autres tissus, se développe alors sous l'influence d'autres facteurs ; à moins encore qu'il ait déjà une plus grande sensibilité que les autres tissus à l'action de la STH. D'ailleurs il existe probablement des relations assez étroites entre l'hypophyse et le thymus, comme semblent le montrer notamment les expériences de PIERPAOLI et SORKIN (I967). Ces auteurs ont constaté que l'administration à des souris d'un sérum anti-hypophyse préparé à partir de lapins injectés avec des extraits hypophysaires de souris, provoque une forte régression du thymus, de même que l'hypophysectomie. D'autre part la thymectomie entraîne des modifications au niveau des cellules acidophiles de l'hypophyse.

Quoi qu'il en soit, nous observons une régression du thymus à partir du stade où différents organes vont subir une accélération rapide. On peut même remarquer sur notre graphique (fig. Ir) que cette régression est plus accusée entre les stades 3 et 4 , où les autres organes subissent une poussée rapide, plus lente ensuite où les organes reprennent eux-mêmes une croissance plus lente. Cette observation s'accorderait assez bien avec l'un des rôles que l'on attribue au thymus, faute d'en avoir une connaissance exacte : celui de réserve de nucléoprotéines à utiliser notamment durant les étapes où survient une " poussée " de croissance.

\section{c) Liaisons entre certains organes.}

La remarque précédente suggère d'autre part que si la recherche des périodes où se produisent une série de changements d'allométrie peut révéler des stades physiologiquement importants, l'examen comparé de l'évolution même de la croissance relative de différents organes peut éventuellement révéler aussi des liaisons d'ordre physiologique insoupçonnées.

Il est bien évident en effet qu'aucun organe n'évolue de façon tout à fait indépendante des autres et de l'ensemble corporel, mais les liaisons qui existent entre eux ne se traduisent pas forcément par une évolution comparable sur le plan pondéral. Souvent au contraire les uns accélèrent leur croissance pendant que d'autres la ralentissent, et ce fait même peut avoir éventuellement une signification, comme cela a été suggéré dans le cas du thymus.

Il peut cependant arriver qu'un certain parallélisme d'évolution pondérale traduise entre diverses parties une liaison organique plus profonde, répondant à certaines nécessités du développement. Ainsi le rapprochement des graphiques de croissance ralative des estomacs, des muscles de la tête et du maxillaire inférieur, reproduits figure 9 , présente une certaine signification. Les courbes d'évolution du maxillaire inférieur et des muscles de la tête ne ressemblent pas du tout à celles des grands tissus correspondants, os et muscles. Par contre, elles se rapprochent très bien de celles des estomacs ou de l'ensemble du tube digestif.

La brusque accélération de la capacité stomacale, qui débute au stade 3 , doit nécessairement s'accompagner d'un rapide accroissement de la capacité de broutage ; 
d'où le développement plus rapide, parmi les os, du maxillaire inférieur, et parmi les muscles de ceux de la tête, dont les muscles de la mastication sont les plus importants.

\section{CONCIUSION}

La présentation du déroulement simultané, entre la naissance et I6o jours, de la croissance relative des principaux tissus et organes de 1'Agneau, nous a permis de préciser certaines particularités et étapes du développement, tout en confirmant et en complétant quelques connaissances déjà classiques.

Cette étude a d'autre part permis de mettre en évidence, par l'intermédiaire de divers changements d'allométrie, l'existence de stades intéressants du point de vue physiologique, en particulier le stade 3 aux environs de 25 jours et ro $\mathrm{kg}$ de poids vif, qui apparaît comme un point critique de la croissance, point de départ d'un certain nombre de remaniements internes dans le développement. De tels stades pourraient être pris comme repères en vue de recherches plus approfondies sur les mécanismes de la croissance relative et le rôle de divers facteurs, notamment des facteurs endocriniens.

Ainsi en particulier, aux époques où ont été constatées des différences d'évolution des tissus gras selon le sexe et selon les lieux de dépôt, les facteurs déterminants de ces divergences peuvent êtr€ recherchés. Leur connaissance présenterait d'ailleurs un évident intérêt d'ordre zootechnique tout autant que biologique.

D'autre part la mise en évidence d'organes ou d'unités anatomiques dont l'évolution au cours d'une assez longue période pourrait véritablement refléter celle d'un tissu ou d'un appareil entiers (muscles, squelette, tube digestif, etc.) rendrait service à de nombreux chercheurs qui ne peuvent pas toujours envisager des dissections complètes. Le choix de tels organes de référence nécessite cependant une analyse plus détaillée que celle que nous avons présentée ici, où nous avons fait observer que certaines unités évoluent de façon sensiblement différente de celle du tout.

De même une analyse plus poussée pourrait éventuellement permettre de découvrir, à travers des évolutions parallèles ou inverses de certains organes, dont nous avons donné des exemples, des liaisons d'ordre physiologique qui ne sont pas toujours apparentes dans des études partielles.

Enfin si l'on veut pouvoir comparer d'une manière précise différentes races ou l'influence de diverses conditions d'élevage sur le devenir des animaux, il nous paraît indispensable de posséder, grâce à des investigations détaillées portant sur le plus grand nombre possible de parties corporelles, une véritable échelle de référence de la croissance relative, connue dans des conditions bien déterminées. C'est ce que nous avons voulu commencer à faire par ce travail.

De telles études, permettant notamment de mieux connaître certaines caractéristiques du développement propres à chaque espèce voire à chaque race, de mieux préciser certains âges physiologiques auxquels ces espèces ou races sont comparables, d'approfondir d'une manière générale la connaissance de la physiologie de la croissance, nous paraissent en effet nécessaires à la maîtrise de futures applications zootechniques. 


\title{
SUMMARY
}

\author{
RELATIVE, WEIGHT GAINS OF TISSUES AND ORGANS IN MALE \\ AND FEMALE MERINOS D'ARLES NEW BORN IAMBS
}

Previous investigations on relative growth in the lamb have been inconclusive and difficult to compare because of differences in experimental conditions. This paper reports investigations on relative growth of tissues and organs in Mérinos d'Avles lambs under determined growth conditions.

Seven growth stages from birth to I 60 days of age were investigated as 7 batches of 2 male and 2 female lambs each (fig. I, table $2 b$ ). The animals were dissected using a technique previously mentioned and substantiated in this paper. Spæcial precaution was taken to prevent too great evaporation losses ( 3 per cent of live weight on the average).

A descriptive study of relative weight growth of tissues and organs was tabularized as guaphs of logarithmic coordinates (fig. 5 to 12 ). The allometry coefficients of each tissue or organ were calculated using the regression curves of $\log y$ on $\log x$ with primary equations as follows : $\log y$ $=\alpha \log x+\log b$ ( $x=$ empty body weight; $y=$ weight of organ) (tables $4,5,6$ ). When a change in allometry occured adjustment for two successive regresion curves corresponding to minimum residual variance was taken into account (table 6$)$.

Our results are as follows :

I. At stages $\mathrm{I}, 2,3$ (birth to ro $\mathrm{kg}$ live weight), significantly different increasing allometry coefficients were found for brain, spinal cord, total bone and blood, muscles and organs, fat tissue and fat depots (table 4, fig. 5). However, some individual parts deviate from the usual sequence of corresponding tissues (bottom of table 4 ).

2. Relative growth up to stage 3 ( $10 \mathrm{~kg}$ live weight) is irrespective of sex. From stage $4 \mathrm{on}$, sex exerts a differential effect on some tissues and organs. With differences depending on location, femalc fat tissue developed more than the male : the latter was depressed or plateaued at stages 3 to 5 ( 9 to $15 \mathrm{~kg}$ live weight (fig. 8 and $8 \mathrm{~b}$ ). Male total bone on the contrary was more developed than the female. After stage 3, total organ weight was higher in the male (fig. $6 \mathrm{c}$ ). Between ro and $30 \mathrm{~kg}$ live weight, female carcass efficiency was slightly higher (fig. 14). Differences in gonad development (as shown in fig. 7) are discussed.

3. Some organs seem to develop irrespective of relative growth pattern (fig. 9). Some show constant growth rates corresponding to one allometry curve (fig. Io). However, most of them exhibit alterations of some sort at various stages, $i$. e. change in speed, translation or delay of the allometry curve (fig. 12). Endocrine glands belong to either category (fig. I I and I2).

The highest change frequency was observed at stages 3-4 (day $25-5$ o or 9 to $12 \mathrm{~kg}$ empty body weight) Stage 3 therefore appears as a critical growth stage.

A synoptical diagram (fig. I 3). summarizes the relative developments of tissues and organs.

4. Our results are discussed and various hypotheses can be tentatively postulated. Our comparisons point out presumptive evidence for the existence of a stable endocrine balance identical for both sexes up to stage 3 . Prior to stage 4 lambs seem to be little responsive to STH. Then alterations in this balance are liable to happen calling into action pituitary stimulating hormones at rates depending on sex. A new balance can then establish, with a subsequent stability in relative growth.

\section{RÉFÉRENCES BIBL,IOGRAPHIQUES}

Bénévent M., I968. Guide pour la dissection et l'identification des principaux muscles de la carcasse chez le mouton. Ann, Biol, anim. Biochim, Biophys., 8, 147-r93.

Birge C. A., Peake G. T., Mariz I. K., Daughaday W. H., r967, Radioimmunoassayable growth hormone in the rat pituitary gland : effects of age, sex and hormonal state. Endocrinology, 81, r95-204.

Boccard R., Dumont B.-L., Lefebvre J., r962. Étude de la production de la viande chez les ovins. V. Note sur la croissance relative des régions corporelles de l'agneau. Ann. Zootech., 11, $257-262$.

Boccard R., Duplan J.-M., I96I. Étude de la production de la viande chez les ovins. III. Note sur l'influence de la vitesse de croissance sur la composition corporelle des agneaux. Ann. Zootech., 10, $3 I-38$. 
Boccard R., Le Guelte P., Arnoux J., I964. Influence de la vitesse de croissance sur la valeur des coefficients d'allométrie des tissus corporels de l'agneau. C. R. Acad. Sci. Paris, 258, I9o8-rgog.

Cantier J., Vezinhet A., Rouvier R., Dauzier L., I969. Allométrie de croissance chez le lapin. (Oryctolagus cuniculus). Ann. Biol, anim. Biochim. Biophys., 9, 5-39.

CORDESSE R., I968. Importance de la multiplication cellulaire au cours de la croissance post-natale du tissu musculaire du lapin commun. D. E. A. Fac. Sci. Montpellier.

Courot M., I962. Développement du testicule chez l'agneau. Établissement de la spermatogenèse. Annls. Biol. anim. Biochim. Biophys., 2, 25-4r.

Cramer D. A., Marchello J. A., r964. Seasonal and sex patterns in fat composition of growing lambs. J. Anim. Sci., 23, IOOz-IOIO.

Durand G., Fauconneau G., Penot E., I965. Étude biochimique de la croissance de l'intestin grêle, du foie et de la carcasse du rat ; rôles respectifs de la multiplication et du grandissement cellulaires. Annls. Biol. anim. Biochim. Biophys., 5, 163-187.

Durand G., Fauconneau G., Penot E., ig67. Croissance des tissus du rat et réduction de l'apport énergétique de la ration; influence sur la teneur en acides nucléiques. Annls. Biol. anim. Biochim. Biophys., \%, I45-I55.

Elsley F. N. H., McDonald I., Fowler V. R., I964. The effect of plane nutrition on the carcasses of pigs and lambs when variations in fat content are excluded. Anim. Prod., 6, I4I-I54.

Flamant J.-C., Boccard R., I966. Estimation de la qualité de la carcasse des agneaux de boucherie (mise au point bibliographique). Ann. Zootech, 15, 89 -I 13.

Fourie P. D., I962. Growth and development of sheep. I. A carcass dissection technique. $N Z$. Jlagric. Res., 5, r9o-222.

Goldspink G., I962. Studies on postembryonic growth and development of skeletal muscle. Proc. $R$. Ir. Acad. ser. B., 62, I35-I50.

Gould S. J., 1966. Allometry and size in ontogeny and phylogeny. Biol. Rev., 41, 587-640.

HAMmond J., I932. Growth and the development of mutton qualities in the sheep. Pp. 597, Oliver and Boyd, Edinburgh.

Hikami Y., Mizuno T., I965. The effects of growth on the cell diameter, cell number and nucleic acid content of skeletal muscles of chicks. Jap. J. Zootech. Sci., 36, 384-39r.

Huxlex J. S., I932. Problems of relative growth. Pp. 276, Methuen, London.

JAckson T. H., 1967. The allometric relationships between carcass muscle and carcass bone in scottish blackface sheep. Anim. Prod., 9, 531-533.

JoUBERT D. M., I956. An analysis of factors influencing post-natal growth and development of the muscle fibre. J. agric. Sci. Camb., 47, 59-102.

LAIRD A. K., I965. Dynamics of relative growth. Growth., 29, 249-263.

LARGE R. V., I964. The development of the lamb with particular reference to the alimentary tract. Anim. Prod., 6, I69-I78.

Marchello J. A., Cramer D. A., Sutherland., Ig6r. Variations in lamb fat from different areas of the carcass. J.Anim. Sci., 20, 680 (Abstr.).

McMeekan C. P., I940. Growth and development in the pig, with special reference to carcass quality characters. J. agric. Sci. Camb., 30, 276, 387, 511 .

McMeeKan C. P., r96o. Principles of animal production, 80-98, Whitcombe and Tombs, Londres.

Palsson H., I955, in : HAMmond J. Progress in the physiology of farm animals, vol. 2, 430-542, Butterworths, Londres.

Palsson H., Verges J. B., I952 $a$. Effects of the plane of nutrition on growth and the development of carcass quality in lambs. I. The effects of high and low planes of nutrition at different ages. J.agric. Sci. Camb., 42, I-92.

Palsson H., Verges J. B., $1952 b$. Effects of the plane of nutrition on growth and the development of carcass quality in lambs. II. Effects on lambs of $30 \mathrm{lb}$. carcass weight. J. agric. Sci. Camb., 42, 93-I39.

Palsson H., Verges J. B., I965. The effect of the plane of nutrition on the shape of the ribs in lambs. J. agric. Sci. Camb., 64, 247-250.

Pierpaoli W., Sorkin E., 1967. Relationship between thymus and hypophysis. Nature, 215, 834-837.

Prud'hon M., Denoy I., Dauzier L., Desvignes A., ig66. Étude des résultats de six années d'élevage des brebis Mérinos d'Arles du domaine du Merle. I. Le contrôle des ruts et sa validité. Ann. Zootesh., 15, I23-I33.

Prud'hon M., Denoy I., Desvignes A., ig68. Étude des résultats de six années d'élevage des brebis Mérinos d'Arles du domaine du Merle. III. La mortalité des agneaux. Ann. Zootech., 17, I59-168.

Russel A. J. F., Barton R. A., r967. Bone muscle relationships in lamb and mutton carcasses. $J$. agric. Sci. Camb., 68, I87-190.

Sefвeck R. M., I968. A dissection study of the distribution of tissus in lamb carcasses. Proc. Aust. Soc. Anim. Prod., $7,297-302$.

Skinner J. D., Booth W. D., Rowson L. E. A., Karg H., I968. The post-natal development of the reproductive tract of the Sulffolk ram, and changes in the gonadotrophin content of the pituitary. $J$. Reprod. Fert., 16, 463-477. 
Teissier G., I934. Dysharmonies et discontinuités dans la croissance. Actualités Scientifiques et Industrielles, $\mathrm{n}^{\circ} 95, \mathrm{pp} .39$, Hermann, Paris.

Vezinhet A., I968 a. Effet de l'hypophysectomie sur la croissance pondérale de l'agneau. C. R. Acad. Sci. Paris, 266, 388-390.

Vezinhet A., 1968 b. Effet de l'hypophysectomie sur la croissance pondérale du lapin. C. R. Acad. Sci. Paris, 266, 2348-2351.

Vezinhet A., 1969. Effets de l'hypophysectomie et d'un traitement chronique à l'hormone somatotrope sur l'évolution pondérale du thymus de l'agneau. C. R. Soc. Biol., 163, 2387-239o.

Vezinhet A., DAUzIER L., I970. Influence de traitements à l'hormone somatotrope bovine sur la croissance pondérale d'agneaux normaux ou hypophysectomisés. Ann. Biol. anim. Biochim. Biophys., $10,5-13$.

Walker D. E., Mc Meekan C. P., 1944. N. $Z$. Jl. Sci. Technol. ser. A., 26, 5I-I 20. Cités par Palsson H., 1955. in : Hammond J., Progress in the physiology of farm animals, vol. 2, 430-542, Butterworths, Londres.

Walker D. G., Asling C. W., Simpson M. E., Li C. H., Evans H. M., 1952. Structural alterations in rats hypophysectomized at six days of age and their correction with growth hormone. Anat. Rec., 114, I9-47.

WALlaCE C., I953. Observations on mammary development in calves and lambs. J. agric. Sci. Camb., 43, 4I3-42I.

WALlace L. R., 1948. The growth of lambs before and after birth in relation to the level of nutrition. Parts II and III. J. agric. Sci. Camb., 38, 243, 367.

Wardrop I. D., Coombe J. B., x96o. The post-natal growth of the visceral organs of the lamb. $J$. agric. Sci. Camb., 54, I40-I44.

Wardrop I. D., Coombe J. B., 196r. The development of rumen function in the lamb. A ust. J.agrc., Res., 12, 66r-680.

WeIL R., I965. Pituitary growth hormone and intermediary metabolism. I. The hormonal effect on the metabolism of fat and carbohydrate. Acta. Endocr. Copenh., 49, suppl. 98. 